\title{
GEOPHYSICS $^{\odot}$
}

\section{Extraction of the tomography mode with non-stationary smoothing for full-waveform inversion}

\begin{tabular}{|r|l|}
\hline Journal: & Geophysics \\
\hline Manuscript ID & GEO-2018-0586.R1 \\
\hline Manuscript Type: & Technical Paper \\
\hline Keywords: & full-waveform inversion, inversion, reflection \\
\hline Area of Expertise: & Seismic Inversion \\
\hline &
\end{tabular}

SCHOLARONE $^{\text {m }}$
Manuscripts 
1 Extraction of the tomography mode with non-stationary smoothing for full2 waveform inversion

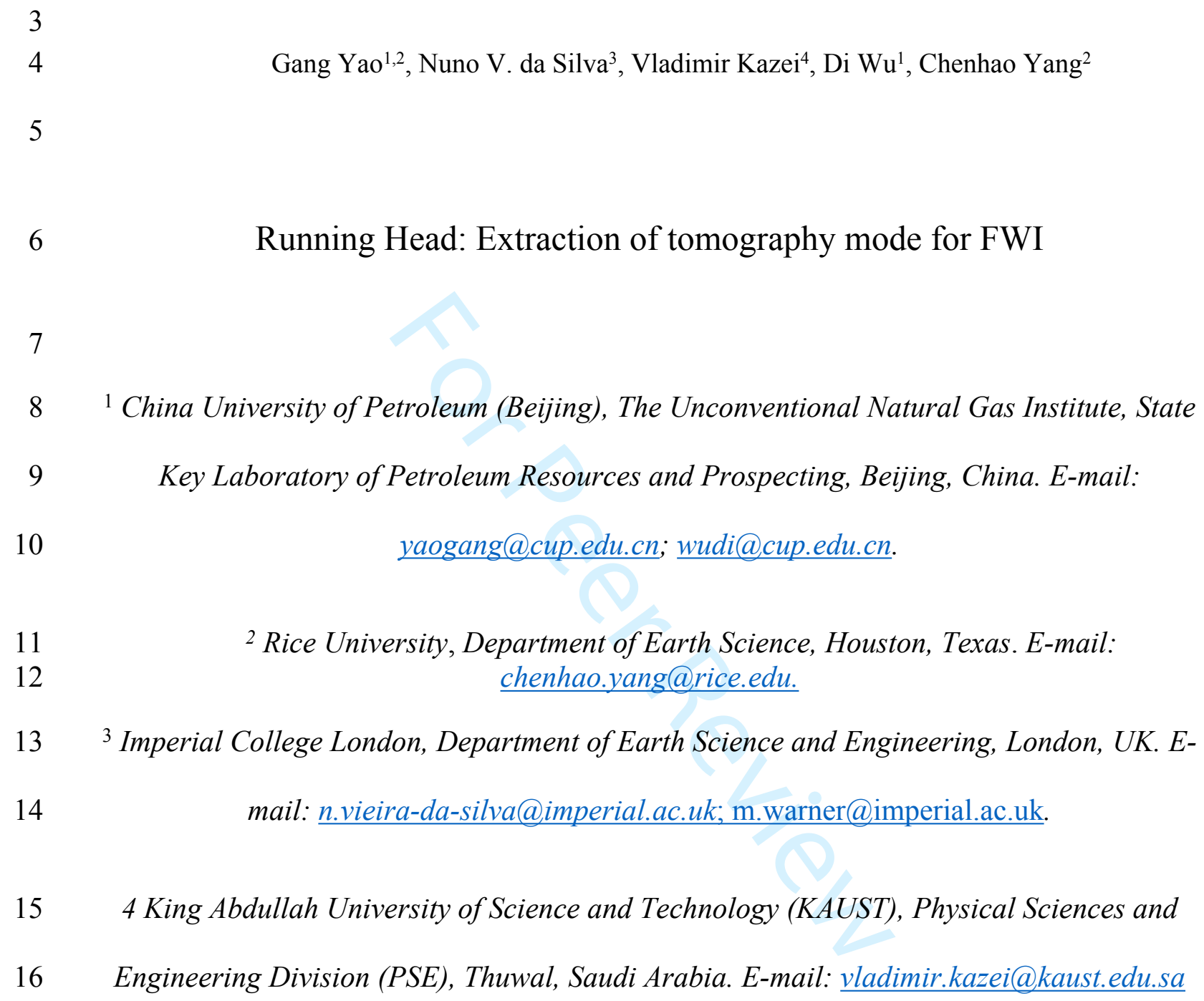
Key Laboratory of Petroleum Resources and Prospecting, Beijing, China. E-mail: mail:n.vieira-da-silva@imperial.ac.uk;m.warner@imperial.ac.uk.

154 King Abdullah University of Science and Technology (KAUST), Physical Sciences and 16 Engineering Division (PSE),Thuwal, Saudi Arabia.E-mail: vladimir.kazei@,kaust.edu.sa

${ }^{2}$ Rice University, Department of Earth Science, Houston, Texas. E-mail: chenhao.yang@rice.edu.

${ }^{3}$ Imperial College London, Department of Earth Science and Engineering, London, UK. E- 
ABSTRACT

Full-waveform inversion (FWI) includes both migration and tomography modes. The

21 tomographic component of the gradient from reflection data is usually much weaker than the migration component. In order to use the tomography mode to fix background velocity errors, it is necessary to extract the tomographic component from the gradient. Otherwise, the inversion will be dominated by the migration mode. We propose a method based on nonstationary smoothing to extract the tomographic component from the raw gradient. By analyzing the characteristics of the scattering angle filtering, the wavenumber of the tomographic component at a given frequency is seen to be smaller than that of the migration component. Therefore, low-wavenumber-pass filtering can be applied to extract the tomographic component. The low-wavenumber-pass smoothing filters are designed with 30 Gaussian filters that are determined by the frequency of inversion, the model velocity, and the 31 minimum scattering angle. Thus, this filtering is non-stationary smoothing in the space domain. Since this filtering is carried out frequency by frequency, it works naturally and efficiently for FWI based on frequency-domain modeling. Furthermore, as the maximum opening angle of 34 the reflections in a typical acquisition geometry is much smaller than the minimum scattering angle for the tomographic component, which is generally set to $160^{\circ}$, there is a relatively large 36 gap between the wavenumbers of the tomographic and migration components. In other words, the non-stationary smoothing can be applied once to a group of frequencies for time-domain FWI without leaking the migration component into the tomographic component. Analyses and numerical tests show that two frequency groups are generally sufficient to extract the 40 tomographic component for the typical frequency range of time-domain FWI. The numerical 41 tests also demonstrate that the non-stationary smoothing method is effective and efficient at extracting the tomographic component for reflection waveform inversion. 
Full-waveform inversion (FWI) is a promising technique for recovering subsurface parameters, including the P-wave and S-wave velocities, in both exploration geophysics (e.g.,

Virieux and Operto, 2009; Warner et al., 2013; Cheng et al., 2016; da Silva et al., 2016, 2019;

Cheng et al., 2017; Yao et al., 2019) and global seismology (e.g., Zhu et al., 2012; Chen et al., 2015; Tao et al., 2017). FWI includes both migration and tomography modes (Mora, 1989).

The migration mode moves and stacks reflection events at their place of origin in order to recover the impedance perturbation, which is missing from the starting model. This migration mode helps FWI to achieve its maximum Rayleigh resolution, i.e., a quarter of the wavelength of the maximum frequency in the record (Kallweit and Wood, 1982). This quarter wavelength resolution means that the minimum wavelength of the image from FWI is half the minimum wavelength of the wavefields (Pratt, 1999; Virieux and Operto, 2009). The tomography mode fixes the background velocity errors along the wave paths inside the first Fresnel zone, the width of which defines the maximum resolution of the tomography mode (Williamson, 1991). Although the tomography mode has a much lower resolution than the resolution limit of the migration mode, it fixes the background velocity errors by improving the focus of the migration mode. The gap between the resolution limits of the two modes can be filled by inverting wideangle reflection and broadband data (Virieux and Operto, 2009; Baeten et al, 2013).

Refraction data, or diving waves, contribute to the tomography mode, whereas reflection data contribute to both modes. The tomographic component in the gradient from reflection data is typically much weaker than the migration component. Therefore, it is necessary to separate them, especially for reflection waveform inversion (RWI), which aims to recover the 65 background velocity using reflection data. Without emphasizing the tomography mode, RWI 66 is dominated by the migration mode and acts like a least-squares reverse-time migration 
67 (LSRTM), which recovers the interfaces rather than the background velocity. A more detailed analysis can be found in Figure 1 of Yao and $\mathrm{Wu}(2017)$.

Several methods exist for the separation of the two modes. All these methods separate the modes by splitting the raw gradient into its tomographic component and its migration component.

Born modeling decomposes the full wave equation into two wave equations, one which generates the incident wavefield and one which produces the scattered wavefield. As a result, the gradient is obtained by pairwise correlation of four wavefields, namely, the incident source wavefield, the scattered source wavefield, the incident residual wavefield, and the scattered

residual wavefield. The tomographic component is formed by cross-correlating the scattered wavefields with the incident wavefields; the migration component is generated by crosscorrelating the incident wavefields from the source and residual wavefields (Xu et al., 2012;

Yao et al., 2014; Zhou et al. 2015; Sun et al., 2016; Audebert and Ortiz-Rubio, 2018). The scattered wavefield with the split wave equations contains both back-scattered and forwardscattered waves, which can be observed clearly with the increase of propagation time.

Consequently, the extracted tomographic component contains some leakage from the migration component.

The method of up-down wavefield separation shares the same principle as the Born modeling method, but uses either the Fourier transform or the Hilbert transform to separate the scattered wavefield from the incident wavefield (Wang et al., 2013; Irabor and Warner, 2016;

$87 \mathrm{Wu}$ and Alkhalifah, 2016; Lian et al., 2018). One limitation is that this method cannot distinguish the scattered waves from the incident waves when both waves propagate along the 89 same direction. A schematic illustration of this can be found in Figure 12 of Liu et al. (2011). 
90 Consequently, strong leakage of the migration component into the tomographic component occurs. The leakage may lead to incorrect updates in RWI (Wang et al. 2018).

Source and residual waves, which contribute to the tomographic component, have an opening angle of around $180^{\circ}$. By contrast, the migration component is formed from waves that have much smaller opening angles. A local slant stack can be used to measure the opening angles in order to distinguish the two components (Xie, 2015). Similarly, scattering angle filtering is an effective and versatile method of separating the two types of components from 97 the raw gradient (Wu and Alkhalifah, 2015, 2017; Kazei et al., 2016; Yao et al., 2018). However, scattering angle filtering significantly increases the cost of the inversion.

The inverse scattering and energy norm (Rocha et al., 2016) imaging conditions can be considered as special cases of scattering angle filtering, in which the filter has a cosine-square 101 transition band from $0^{\circ}$ to $180^{\circ}$. These conditions are sufficient for extracting the migration component (Whitmore and Crawley, 2012; Fang et al., 2018; Rocha et al., 2018; Yang and Zhang, 2018). However, due to the wide transition band, modifications such as adaptive weighting are required to achieve an effective extraction of the tomographic component (Ramos-Martinez et al., 2016).

Here, we analyze the characteristics of scattering angle filtering and propose a method of 107 non-stationary smoothing with Gaussian filters to extract the tomographic component from the 108 raw gradient. Compared to a conventional FWI (Tarantola, 1984; Pratt, 1999) implementation, 109 the computational cost of the non-stationary smoothing is trivial, though it needs one extra 110 backpropagation for time-domain FWI in a typical frequency range (i.e., $<20 \mathrm{~Hz}$ ). Using 111 numerical examples, we demonstrate that this method is effective and efficient for the 112 extraction of the tomographic component and for applications to RWI. 


$$
\phi=\frac{1}{2}\left(\mathbf{d}-\mathbf{d}_{0}\right)^{T}\left(\mathbf{d}-\mathbf{d}_{0}\right)=\frac{1}{2} \delta \mathbf{d}^{T} \delta \mathbf{d},
$$

116 where $\mathbf{d}$ and $\mathbf{d}_{0}$ represent the predicted and observed data, respectively, and $\delta \mathbf{d}$ is the residual.

117 If the P-wave velocity is the inversion parameter, then the gradient is expressed as

$$
\frac{\partial \phi}{\partial v}=g(\mathbf{x})=-\frac{2}{v^{3}} \sum_{t} \&_{s}(\mathbf{x}, t) \&(\mathbf{x}, t)
$$

119 where $p_{s}$ and $p_{r}$ represent the source wavefield and the backpropagated residual wavefield, 120 respectively, $v$ denotes the P-wave velocity, and '.' means the first-order time derivative. For 121 a constant velocity model, the wavenumber-domain counterpart of the gradient expressed in 122 equation 2 can be written as

$$
g /(\mathbf{k})=-\frac{2}{v^{3}} \sum_{t} \frac{\beta}{s}\left(\mathbf{k}_{s}, t\right) * \frac{8}{\rho}\left(\mathbf{k}_{r}, t\right)
$$

124 where ' $\sim$ ' denotes the counterpart of wavefields or gradient in the wavenumber domain, $\mathbf{k}_{s}$, $125 \mathbf{k}_{r}$, and $\mathbf{k}$ are the wavenumber vectors of the source wavefield, the residual wavefield, and 126 the gradient, respectively, and ' $*$ ' represents a multi-dimensional wavenumber convolution.

127 The wavenumber vectors of the gradient satisfy the relationship

$$
\mathbf{k}=\mathbf{k}_{s}+\mathbf{k}_{r} .
$$

129 The detailed derivation of equation 4 can be found around equation 7 of Yao et al. (2018). In 130 acoustic isotropic media, for a frequency, $\omega$, and a velocity, $v$, 


$$
\left|\mathbf{k}_{s}\right|=\left|\mathbf{k}_{r}\right|=\frac{\omega}{v} .
$$

132 According to equations 4 and 5, the magnitude of the gradient's wavenumber can be 133 calculated using

$$
|\mathbf{k}|=\frac{2 \omega}{v} \cos \frac{\alpha}{2},
$$

135 where $\alpha$ is the opening angle of the source and receiver wavenumber vectors.

We know that if the source wavefield propagates opposite the residual wavefield, then the

137 cross-correlation of the two wavefields contributes to the tomographic component of the 138 gradient. In other words, the tomographic component is formed by the source and residual contribute to the migration component of the gradient. One effective method of separating the

141 two components is scattering angle filtering based on equation 6 (Alkhalifah, 2015; Wu and 142 Alkhalifah, 2015, 2017). As the tomographic component comes from greater scattering angles 143 than the migration component, equation 6 implies that the tomographic component has a 144 smaller wavenumber than the migration component at a given frequency and velocity (Figure 145 1). In the figure, the wavenumbers of the migration component sit in the light-gray outer ring, 146 and the wavenumbers of the tomographic component are located inside the dark-gray inner 147 circle. The transition zone lies between the two. Therefore, we extract the tomographic 148 component by using low-wavenumber-pass filtering, which is equivalent to a process of 149 smoothing in the space domain.

150 To extract the tomographic component from one element of the raw gradient at one 151 frequency, we begin by computing the cut-off wavenumber from 


$$
k_{\text {tomo_max }}=\frac{2 \omega}{v} \cos \frac{\alpha_{\min }}{2},
$$

153 where $\omega$ is the frequency of inversion, $v$ is the model velocity at this element, and $\alpha_{\min }$ is the

154 selected minimum opening angle. Next, we design a space-domain smoothing filter that is 155 equivalent to having a preset damped amplitude at the cutoff wavenumber, $k_{\text {tomo_max }}$. Then, we 156 convolve this filter with the raw gradient to extract its tomographic component at the element 157 being processed for the frequency of inversion. Finally, we repeat this process for all the 158 elements and frequencies. Thus, the smoothing process is non-stationary.

159 In this paper, we design the space-domain smoothing filter by using the Gaussian function, 160 which is defined as

161

$$
G(x)=\frac{1}{\sqrt{2 \pi} \sigma} e^{-\frac{x^{2}}{2 \sigma^{2}}}
$$

162 its Fourier domain expression is

$$
\&(\phi k)=e^{-\frac{k^{2}}{2 \sigma_{k}^{2}}}
$$

164 where $\sigma$ and $\sigma_{k}$ denote the standard deviations in the space domain and in the Fourier domain, 165 respectively, and $k$ is the wavenumber (units in $1 / \mathrm{m}$ ). The standard deviations determine the 166 shape of the Gaussian functions in each respective domain. The Fourier transform pair used for 167 equations 8 and 9 is listed in APPENDIX A. The two standard deviations share the relationship 168 of

$$
\sigma \sigma_{k}=\frac{1}{2 \pi}
$$


170 Thus, the cut-off wavenumber $k_{\text {tomo_max }}$ can be calculated using equation 7 with the

171 frequency of inversion, the velocity of the element being processed, and the minimum opening 172 angle, $\alpha_{\min }$, which is set to $160^{\circ}$ for the tests in this paper. To define the Fourier-domain 173 Gaussian function, we set the amplitude value, $\beta$, to 0.3 for the cut-off wavenumber $k_{\text {tomo } \max }$ 174 (Figure 2a). According to equation 9, the standard deviation, $\sigma_{k}$, can be computed with

$$
\sigma_{k}=\frac{k_{\text {tomo_max }}}{\sqrt{-2 \log \beta}} .
$$

Then, the standard deviation, $\sigma$, can be determined from equations 10 and 11 as

$$
\sigma=\sqrt{\frac{-\log (\beta)}{2 \pi^{2} k_{\text {tomo-max }}^{2}}} .
$$

Finally, the Gaussian filter is obtained using equation 8. Since a Gaussian function has infinitely long tails, we truncate its tails by setting the truncation factor, $\gamma$, to 0.01 (Figure $2 b$ ).

180 Consequently, the effective radius of the Gaussian filter is

$$
l=\sqrt{-2 \sigma^{2} \log (\gamma)},
$$

182 which is illustrated in Figure 2b.

According to equation 7 , we extract the tomographic component by smoothing the raw

184 gradient frequency by frequency. This approach is convenient for FWI based on frequencydomain modeling. However, if we directly apply this approach to FWI based on time-domain modeling, we need to Fourier transform the wavefields in the whole model from the time

187 domain into the frequency domain. This transform leads to a significant increase in storage and 188 computational cost. As illustrated in Figure 1, there is a transition zone between the 
189 tomographic component zone and the migration component zone in the wavenumber domain

190 because the maximum opening angle for the migration component is smaller than the minimum

191 opening angle for the tomographic component in a typical acquisition geometry. The transition

192 zone implies that the smoothing process based on one reference frequency can be applied on

193 the raw gradient generated by a group of frequencies as long as the minimum wavenumber of

194 the migration component of the lowest frequency is bigger than the maximum wavenumber of 195 the tomographic component of the highest frequency. We give additional details about the 196 strategy of the frequency grouping in the next section.

\section{One-Source-One-Receiver Geometry}

The first example is on a two-layer model (Figure 3a). In this test, we used one-source-

200 one-receiver geometry. The source and the receiver are located at a distance of $2 \mathrm{~km}$ and $6 \mathrm{~km}$, 201 respectively, and a depth of $2.5 \mathrm{~km}$. More detailed information about the model and the geometry can be found in the caption of Figure 3. The source wavelet is a bandlimited spike, which has a flat spectrum from $3 \mathrm{~Hz}$ to $20 \mathrm{~Hz}$ and is shown in Figure $3 \mathrm{~b}$. This spectrum covers the typical frequency range of current FWI applications. In this test, we only used reflections

205 to form the gradient. The raw gradient of the first iteration is shown in Figure 3a. The migration 206 component is represented by the ellipse in the figure; the remainder of the gradient is the 207 tomographic component, which contains the information required for the background update.

208 The wavenumber spectrum of the raw gradient is shown in Figures 3c-d, where the spectrum 209 of the tomographic component appears as four narrow ellipses symmetrically located around 210 the origin; the remaining circular shapes are the migration component. As can be seen, the two 211 components are mixed in the wavenumber domain. 
212 To extract the tomographic component, we apply non-stationary smoothing to the raw

213 gradient. The cutoff angle is set to $160^{\circ}$. The value of the Gaussian function of the cutoff

214 wavenumber (equation 9) is 0.3 in the Fourier domain. Figure 4a shows that the tomographic

215 component is successfully extracted frequency by frequency. The wavenumber spectrum of the

216 extracted tomographic component is shown in Figure 4b. However, smoothing frequency by

217 frequency requires a Fourier transform on the wavefields; therefore, the computational and 218 storage costs are prohibitive for time-domain FWI.

219 As the discussion at the end of the previous section and the illustration of Figure 1 show, 220 there exists a transition zone between the tomographic component zone and the migration 221 component zone in the wavenumber domain because the maximum opening angle of reflection 222 events is smaller than the cutoff angle. In this case, we set the cutoff angle to $160^{\circ}$. 223 Consequently, if the opening angle of the reflection events is smaller than $140^{\circ}$, which satisfies 224 most real applications, the maximum wavenumber of the tomographic component will be less 225 than half of the minimum wavenumber of the migration component. As a result, we can apply 226 the non-stationary smoothing once on a group of frequencies ranging from half to double the 227 reference frequency.

To test this strategy, we define four frequency groups with reference frequencies of 1.5 $229 \mathrm{~Hz}, 3 \mathrm{~Hz}, 6 \mathrm{~Hz}$, and $12 \mathrm{~Hz}$, which cover the whole frequency range of the data. The reasons for 230 choosing the four reference frequencies in this example are as follows: (1) half of one reference 231 frequency is equal to the immediately lower reference frequency, while double the reference 232 frequency is equal to the immediately higher reference frequency; and (2) the frequency range 233 from half of the smallest reference frequency to double the highest reference frequency covers 234 the frequency bandwidth of the backpropagated residual wavefields. In order to select these 235 frequencies, we filter the residual using the four filters shown in Figure 5a. The transition bands 
236 of the four filters are formed by cosine-square functions, so the summation of the four filtered

237 residuals is identical to the original residual. We do not need to filter the source wavelet or the

238 source wavefield because the zero-lag cross-correlation used to form the gradient removes the

239 frequencies of the source wavefield that do not exist in the filtered residual wavefield. The four

240 residuals yield four sub-gradients. The tomographic components of the sub-gradients were

241 extracted separately by using non-stationary smoothing with the four reference frequencies.

242 Figure $5 \mathrm{c}$ shows the stack of four tomographic components, the wavenumber spectrum of

243 which is shown in Figure 5d. From Figures 5c and 5d, we see that the strategy of using

244 frequency groups is as effective as the means of filtering frequency by frequency.

However, in practice, we have to backpropagate the filtered residuals four times, which

246 is computationally expensive. To reduce the computational cost, we reduce the reference

247 frequencies to only $6 \mathrm{~Hz}$ and $12 \mathrm{~Hz}$ since the wavelet has very weak energy for the frequencies

248 lower than $3 \mathrm{~Hz}$ and higher than $24 \mathrm{~Hz}$. Thus, only one extra backpropagation is sufficient in

249 practice. The two sub-gradients are shown in Figures 5e and 5f, and their wavenumber spectra

250 are depicted in Figures 5h and 5i. The tomographic component is clearly separated in the

251 wavenumber domain. In other words, the tomographic component is extracted successfully

252 with just two frequency groups (Figure 5g). In comparison, using just two frequency groups

253 does not noticeably decrease the quality, except for some weak artifacts between the two 'rabbit

254 ears' indicated by the triangle in Figure $5 \mathrm{~g}$. Fortunately, these weak artifacts (about $5 \%$ in this

255 example) do not noticeably affect the inversion.

If we do not apply the frequency group strategy, the quality of the extracted tomographic 257 component is reduced significantly. For example, when we choose $6 \mathrm{~Hz}$ as the only reference 258 frequency, the non-stationary smoothing removes some high wavenumbers from the 259 tomographic component (Figures 6a and 6c). If the reference frequency is $12 \mathrm{~Hz}$, some low 
260 wavenumbers from the migration component leak into the tomographic component (Figures

$2616 \mathrm{~b}$ and $6 \mathrm{~d})$.

262 From the colorbars in this example, the smoothing process noticeably shrinks the 263 amplitude of the tomographic component but keeps its shape. This correct shape is essential

264 for a successful inversion, while the amplitude loss can be compensated by the step length.

\section{Application to Reflection Waveform Inversion}

266 In the second example, we apply the two-frequency-group strategy to the Marmousi 267 model (Figure 7a) for RWI (Yao et al., 2014; Yao and Wu, 2017). We generate 128 shots using 268 the acoustic wave equation. The source wavelet is a bandlimited spike, which is shown in 269 Figure 8a. It has a flat spectrum between $3 \mathrm{~Hz}$ and $12 \mathrm{~Hz}$ (Figure 8b). The source of the first 270 shot is located at a distance of $187.5 \mathrm{~m}$. The shot interval is $100 \mathrm{~m}$. The maximum offset of 271 each shot is $3.75 \mathrm{~km}$. All the sources and receivers are fixed at a depth of $25 \mathrm{~m}$. An absorbing 272 boundary condition is applied around the whole model. Consequently, the synthesized record simulates the seismic data after multiple removal and deghosting. However, the nonstationary 274 smoothing method also works in the presence of multiples. The one shot record is shown in 275 Figure 9a. The direct arrivals and refractions are removed in the inversion. The muted record 276 corresponding to the shot gather in Figure 9a is depicted in Figure 9b.

We alternated between a migration-like step and a tomography-like step to iteratively update the background velocity model with reflection-only data (Yao et al., 2014; Yao and Wu, 2017). The migration-like step uses the short-offset data to build a temporary model of 280 reflectors, which produce predicted reflection events that match the recorded reflection events 281 inside the short offsets in a least-squares sense. However, the rest of the offsets have relatively 
282 large residuals, which contain information for the background velocity update. Then, the 283 tomography-like step uses all the offsets to update the background.

284 A multiscale strategy (Bunks et al., 1995) is applied in the inversion. The inversion is 285 started from $4 \mathrm{~Hz}$ and increases to $10 \mathrm{~Hz}$ in increments of $1 \mathrm{~Hz}$. Each frequency is extracted 286 by bandpass filtering on the residual centered at this frequency. The filter damps the 287 frequencies lower than the central frequency, fully stopping at $0 \mathrm{~Hz}$; it also damps the 288 frequencies higher than the central frequency, coming to a full stop at 2.5 times the central 289 frequency. Each frequency is inverted for five iterations; therefore, there are 35 iterations in 290 total.

291 The gradients of the tomography-like step from the first iteration are shown in Figure 10.

292 The data from the first iteration are extracted using a band-passed filter centered at $4 \mathrm{~Hz}$. The

293 two reference frequencies used to extract the tomographic component are $2.67 \mathrm{~Hz}\left(=\frac{2 f}{3}\right.$,

294 where $f$ is the central frequency, i.e. $4 \mathrm{~Hz})$ and $5.33 \mathrm{~Hz}\left(=\frac{4 f}{3}\right)$, and their corresponding raw 295 gradients are shown in Figures 10a and 10c, respectively. Their stack is depicted in Figure 10e; 296 it is dominated by the high-wavenumber migration component, which shows the geological 297 structure of Marmousi. In contrast, the tomographic component is much weaker.

To complete the tomography-like step, we apply non-stationary smoothing with the two299 frequency-group strategy to the raw gradients. The output is shown in Figures 10b, 10d, and 300 10f. The extracted tomographic component is very smooth and matches the background trend 301 that faintly appears in the raw gradients, so we conclude that it is very useful for the background 302 velocity update. The colors indicate that the velocity in the left top region should decrease 303 while the velocity in the right part should increase. This coincides with the background trend 304 of the errors in the initial model. The successful extraction can be attributed to the clear 
305 separation of the migration and tomographic components in the wavenumber domain. An 306 illustration is shown in Figure 11.

307 From the spectra shown in Figure 11, we see that in this example, unlike the one-source308 one-receiver example shown in Figures 4-6, non-stationary smoothing does not reduce the 309 amplitude of the tomographic component significantly. This difference between the two 310 examples is mainly due to the high wavenumbers of the tomographic component (i.e. the rabbit 311 ears) in the one-source-one-receiver example being generated by the oscillations of the 312 sidebands of the rabbit ears, which do not include useful information about the background 313 update. These oscillatory sidebands are cancelled out in the Marmousi example by the stack of 314 multi-shots and multi-receivers.

315 If we design the Gaussian filter using a single reference frequency of $4 \mathrm{~Hz}$ or $12 \mathrm{~Hz}$ and 316 a constant velocity of $2700 \mathrm{~m} / \mathrm{s}$, then the process becomes stationary Gaussian smoothing (e.g. 317 Abdullah and Schuster, 2015; Kazei et al., 2016). The results are shown in Figures 10g and 318 10h. Compared with the nonstationary result shown in Figure 10f, the stationary smoothing 319 either filters out some tomographic component (Figure $10 \mathrm{~g}$ ) or leaks some high-wavenumber 320 migration component into the tomographic component (Figure 10h). Thus, stationary 321 smoothing leads to a degradation of the inversion results.

The inverted model after 35 alternating iterations is shown in Figure 12. The RWI result with non-stationary smoothing is shown in Figure 12a. It is a good match to the background trend of the Marmousi velocity model, especially above the top boundary of the thick highvelocity salt layer, which is at a depth of around $2.5 \mathrm{~km}$ to $3 \mathrm{~km}$. In contrast, RWI with stationary smoothing produces low-quality results. The reference frequency of $4 \mathrm{~Hz}$ produces

327 a background model with lower resolution and more errors, for example, in the area at a 328 distance of $8 \mathrm{~km}$ to $10 \mathrm{~km}$ (Figure $12 \mathrm{~b}$ ), because the stationary smoothing removes some high- 
329 wavenumber tomographic component unnecessarily. The reference frequency of $12 \mathrm{~Hz}$ 330 generates a background model full of high-wavenumber artifacts (Figure 12c). This is because

331 the stationary smoothing leaks a significant amount of the migration component into the 332 tomographic component. The leaked migration component prevents an effective background 333 update.

334 Starting from the initial model and the RWI results (Figure 12), we carried out 335 conventional FWI, as introduced by Tarantola (1984), with the reflection data (e.g. Figure 9b) 336 from $4 \mathrm{~Hz}$ up to $10 \mathrm{~Hz}$ with an increment of $1 \mathrm{~Hz}$ (Bunks et al., 1995). Each frequency is 337 inverted for five iterations; therefore, there are 35 iterations in total. We note that each 338 'frequency' means a frequency band centered at this frequency. The inverted models are 339 depicted in Figure 13. From this comparison, we see the RWI model with nonstationary 340 smoothing produces a better final model than the others. This is because the RWI model with 341 nonstationary smoothing produces a good travel time match. This can be seen from the 342 alternating display of a predicted shot gather and its corresponding recorded shot gather in 343 Figure 14. The improved position of reflectors in the stacked migration profiles (Figure 15) 344 and the flatness of two common-image-gathers (CIGs) (Figure 16) also suggest that the RWI 345 model turned out to be kinematically correct.

\section{DISCUSSION}

We choose the Gaussian filter to extract the tomographic component of the gradient

348 because the filter can be computed analytically, i.e., at a negligible computational cost.

349 However, it also unnecessarily suppresses those wavenumbers smaller than the cutoff 350 wavenumber, $k_{\text {tomo } \max }$ (Figure 2a). To mitigate this undesired suppression, the cutoff angle

$351 \alpha_{\min }$ has to be chosen adequately and as small as possible, but without leakage from the 352 migration component. As a rule of thumb, in this paper, we set the cutoff angle to $160^{\circ}$. 
353 Alternatively, we may find a filter that has a narrow transition band, unlike a Gaussian filter. 354 Ideally, we expect this filter to have a simple analytical expression to avoid computing it 355 numerically.

\section{CONCLUSION}

We analyzed the characteristics of scattering angle filtering and found that the

wavenumber of the tomographic component of the raw gradient is smaller than that of its

360 migration component. The tomographic component can, therefore, be extracted by low-

361 wavenumber-pass filtering. We used Gaussian filters to achieve the low-wavenumber-pass

362 filtering. As the Gaussian filters depend on the frequency of the inversion, the model velocity,

363 and the minimum scattering angle, the filtering is a non-stationary smoothing process. This

364 method can be directly applied to FWI based on frequency-domain modeling. As there is a

365 relatively wide gap between the wavenumbers of the tomographic component and the 366 migration component for reflection data, for the time-domain FWI, we divide the residual into 367 several frequency groups. Then, we apply the non-stationary smoothing to each frequency 368 group separately in order to prevent the migration component from leaking into the 369 tomographic component. The two numerical tests demonstrated that the strategy of using just 370 two frequency groups extracts the tomographic component successfully. With this strategy, 371 only one extra backward propagation per iteration is necessary to extract the tomographic mode 372 of the inversion within a typical frequency range.

\section{ACKNOWLEDGMENTS}

374 This research was supported by the National Key Research and Development Program of 375 China (No. 2017YFC1500303), NSFC (Grant No. 41630209), NSF (Grant No. 1547228), 
376 Science Foundation of China University of Petroleum, Beijing (No. 2462018BJC001, No.

377 2462015YJRC012), and the FULLWAVE consortium. The authors would like to thank Editors

378 A. Baumstein and J. Shragge, and four anonymous reviewers, for their comments and 379 suggestions, which helped to improve and clarify the manuscript significantly. Finally, the 380 authors would also like to show gratitude to Veronica E. Tremblay for the proofreading. 


\section{APPENDIX A}

\section{FOURIER TRANSFORM PAIR}

384 The continuous Fourier transform pair used for equations 8 and 9 is

$$
f / \phi k)=\int_{-\infty}^{\infty} f(x) e^{-j 2 \pi x \cdot k} d x
$$

386 and

387

$$
\left.f(x)=\int_{-\infty}^{\infty} f / \phi k\right) e^{j 2 \pi x \cdot k} d k
$$

388 where $x$ denotes distance (unit: meter) and $k$ represents wavenumber (unit: $1 / \mathrm{m}$ ). 


\section{REFERENCES}

Abdullah, A., and G. T. Schuster, 2015, Inverting reflections using full-waveform

393 inversion with inaccurate starting models: 85th Annual International Meeting, SEG, Expanded 394 Abstracts, 1148-1153.

Alkhalifah, T., 2015, Scattering-angle based filtering of the waveform inversion gradients:

396 Geophysical Journal International, 200, no. 1,363-373. doi: 10.1093/gji/ggu379.

Audebert, F., and D. Ortiz-Rubio, 2018, Separation of scales in FWI and RFWI. Filling 398 the gap? Or exploiting it?: 88th Annual International Meeting, SEG, Expanded Abstracts, $399 \quad 1324-1328$.

Baeten, G., J. W. de Maag, R. Plessix, R. Klaassen, T. Qureshi, M. Kleemeyer, F. t. 401 Kroode, and Z. Rujie, 2013, The use of low frequencies in a full-waveform inversion and 402 impedance inversion land seismic case study: Geophysical Prospecting, 61, no. 4, 701-711, 403 doi:10.1111/1365-2478.12010

Bunks, C., F. Saleck, S. Zaleski, and G. Chavent, 1995, Multiscale seismic waveform 405 inversion, Geophysics, 60, no. 5, 1457-1473, doi: 10.1190/1.1443880.

Chen, M., F. Niu, Q. Liu, J. Tromp, and X. Zheng, 2015, Multiparameter adjoint 407 tomography of the crust and upper mantle beneath East Asia: 1. Model construction and 408 comparisons: Journal of Geophysical Research: Solid Earth, 120, no. 3, 1762-1786, 409 doi:10.1002/2014JB011638.

410 Cheng, X., K. Jiao, D. Sun, and D. Vigh, 2016, Multiparameter estimation with acoustic 411 vertical transverse isotropic full-waveform inversion of surface seismic data: Interpretation, 4, 412 no. 4, SU1-SU16, doi:10.1190/INT-2016-0029.1. 
413 Cheng, X., K. Jiao, D. Sun, Z. Xu, D. Vigh, and A. El-Emam, 2017, High-resolution 414 Radon preconditioning for full-waveform inversion of land seismic data, Interpretation, 5, no. 415 4, SR23-SR33, doi:10.1190/INT-2017-0020.1.

416 da Silva, N., A. Ratcliffe, V. Vinje, and G. Conroy, 2016, A new parameter set for 417 anisotropic multiparameter full-waveform inversion and application to a North Sea data set: 418 Geophysics, 81, no. 4, U25-U38, doi: 10.1190/geo2015-0349.1.

da Silva, N., G. Yao, and M. Warner, 2019, Semiglobal viscoacoustic full-waveform 420 inversion: Geophysics, 84, no. 2, R271-R293, doi: 10.1190/geo2017-0773.1.

Fang, X. Z., F. L. Niu, and D. Wu, 2018, Least-squares reverse-time migration enhanced 422 with the inverse scattering imaging condition: Chinese Journal of Geophysics (in Chinese), 61 , no. 9, 3770-3782, doi: 10.6038/cjg2018L0721.

Irabor, K., and M. Warner, 2016, Reflection FWI: 86th Annual International Meeting, 425 SEG, Expanded Abstracts, 1136-1140.

Kallweit, R., and L. Wood, 1982, The limits of resolution of zero-phase wavelets. 427 Geophysics, 47, no. 7, 1035-1046, doi: 10.1190/1.1441367.

Kazei, V., E. Tessmer, and T. Alkhalifah, 2016, Scattering angle-based filtering via 429 extension in velocity: 86th Annual International Meeting, SEG, Expanded Abstracts, 1157430 1162.

Lian, S., S. Yuan, G. Wang, T. Liu, Y. Liu, and S. Wang, 2018, Enhancing low432 wavenumber components of full-waveform inversion using an improved wavefield 433 decomposition method in the time-space domain: Journal of Applied Geophysics, 157, 10-22. 434 doi: 10.1016/j.jappgeo.2018.06.013. 
435 Liu, F., G. Zhang, S. A. Morton, and J. P. Leveille, 2011, An effective imaging condition 436 for reverse-time migration using wavefield decomposition: Geophysics, 76, no. 1, S29-S39, 437 doi: $10.1190 / 1.3533914$.

438 Mora, P., 1989, Inversion = migration + tomography: Geophysics, 54, no. 12, 1575-1586, 439 doi: $10.1190 / 1.1442625$.

440 Pratt, R., 1999, Seismic waveform inversion in the frequency domain, Part 1: Theory and 441 verification in a physical scale model: Geophysics, 64, no. 3,888-901. doi: 10.1190/1.1444597.

442 Ramos-Martinez, J., S. Crawley, K. Zou, A. Valenciano, L. Qiu, N. Chemingui, and A. 443 Long, 2016, A Robust Gradient for Long Wavelength FWI Updates: 25th International 444 Geophysical Conference and Exhibition: ASEG, Expanded Abstracts, 547-551.

445 Rocha, D., N. Tanushev, and P. Sava, 2016, Acoustic wavefield imaging using the energy 446 norm: Geophysics, 81, no. 4, S151-S163.

447 Rocha, D., P. Sava, and A. Guitton, 2018, 3D acoustic least-squares reverse time 448 migration using the energy norm: Geophysics, 83, no. 3, S261-S270, doi: 10.1190/geo2017$449 \quad 0466.1$.

450 Sun, D., K. Jiao, X. Cheng, and D. Vigh, 2016, Reflection-based waveform inversion: 451 86th Annual International Meeting, SEG, Expanded Abstracts, 1151-1156.

Tarantola, A., 1984, Inversion of seismic reflection data in the acoustic approximation: 453 Geophysics, 49, no. 8, 1259-1266, doi:10.1190/1.1441754. a normalized-correlation-coefficient-based misfit function: Geophysical Journal International, 456 210, no. 3, 1517-1524, doi:10.1093/gji/ggx249. 
457 Virieux, J., and S. Operto, 2009, An overview of full-waveform inversion in exploration 458 geophysics: Geophysics, 74, no. 6, WCC1-WCC26, doi: 10.1190/1.3238367.

459 Wang, F., H. Chauris, D. Donno, and H. Calandra, 2013, Taking Advantage of Wave 460 Field Decomposition in Full Waveform Inversion: 75th Conference and Exhibition, EAGE, $461 \quad$ Extended Abstracts, Tu0708.

462 Wang, P., Z. Zhang, Z. Wei, and R. Huang, 2018, A demigration-based reflection full463 waveform inversion workflow: 88th Annual International Meeting, SEG, Expanded Abstracts, $464 \quad 1138-1142$.

Warner, M., and A. Ratcliffe, T. Nangoo, J. Morgan, A. Umpleby, N. Shah, ..., and A. 466 Bertrand, 2013, Anisotropic 3D full-waveform inversion: Geophysics, 78, no. 2, R59-R80, 467 doi:10.1190/geo2012-0338.1.

Whitmore, N., and S. Crawley. 2012, Applications of RTM inverse scattering imaging 469 conditions: 82nd Annual International Meeting, SEG, Expanded Abstracts, 1-6.

Williamson, P., 1991, A guide to the limits of resolution imposed by scattering in ray tomography: Geophysics, 56, no. 2, 202-207. doi: 10.1190/1.1443032.

Wu, Z., and T. Alkhalifah, 2015, Simultaneous inversion of the background velocity and 473 the perturbation in full-waveform inversion: Geophysics, 80, no. 6, R317-R329, 474 doi:10.1190/geo2014-0365.1.

Wu, Z., and T. Alkhalifah, 2016, The optimized gradient method for full waveform 476 inversion and its spectral implementation: Geophysical Journal International, 205, no. 3, 1823477 1831, doi: 10.1093/gji/ggw112. 
478 Wu, Z., and T. Alkhalifah, 2017, Efficient scattering-angle enrichment for a nonlinear 479 inversion of the background and perturbations components of a velocity model: Geophysical 480 Journal International, 210, no. 3, 1981-1992, doi: 10.1093/gji/ggx283.

481 Xie, X., 2015, An angle-domain wavenumber filter for multi-scale full-waveform 482 inversion: 85th Annual International Meeting, SEG, Expanded Abstracts, 1132-1137.

483 Xu, S., D. Wang, F. Chen, Y. Zhang, and G. Lambare, 2012, Full waveform inversion for 484 reflected seismic data: 74th Conference and Exhibition, EAGE, Extended Abstracts, w024.

485 Yang, K., and J. Zhang, 2018, Least-squares reverse time migration with an angle486 dependent weighting factor: Geophysics, 83, no. 3, S299-S310, doi: 10.1190/geo2017-0207.1.

487 Yao, G., M. Warner, and A. Silverton, 2014, Reflection FWI for both reflectivity and 488 background velocity: 76th Conference and Exhibition, EAGE, Extended Abstracts, We E106 48910

490 Yao, G., and D. Wu, 2017, Reflection full waveform inversion: Science China Earth 491 Sciences, 60, no. 10, 1783-1794, doi: 10.1007/s11430-016-9091-9.

492 Yao, G., N. V. da Silva, M. Warner, and T. Kalinicheva, 2018, Separation of migration 493 and tomography modes of full-waveform inversion in the plane-wave domain: Journal of 494 Geophysical Research: Solid Earth, 123, doi: 10.1002/2017JB015207.

495 Yao, G., N. da Silva, M. Warner, D. Wu, and C. Yang, 2019, Tackling cycle-skipping in 496 full-waveform inversion with intermediate data: Geophysics, 1-62, doi: 10.1190/geo2018$497 \quad 0096.1$. 
2

4

5

6

of

498 Zhou, W., R. Brossier, S. Operto, and J. Virieux, 2015, Full waveform inversion of diving $499 \&$ reflected waves for velocity model building with impedance inversion based on scale 500 separation, Geophysical Journal International, 202, no. 3,1535-1554. doi: 10.1093/gji/ggv228.

501 Zhu, H., E. Bozdağ, D. Peter, and J. Tromp, 2012, Structure of the European upper mantle 502 revealed by adjoint tomography: Nature Geoscience, 5, no.7, 493-498, doi:10.1038/ngeo1501. 
Figure 1. Schematic diagram for the relationship between opening angles and wavenumber distribution. $\alpha_{\text {tomo_min }}$ indicates the minimum opening angle for the tomographic component while $\alpha_{\text {mig_max }}$ indicates the maximum opening angle for the migration component.

$507 \quad \mathbf{k}_{s}$ and $\mathbf{k}_{r}$ are the wavenumber of the source and residual wavefields, respectively. They sit on the dashed circle. The tomographic component sits inside the inner dark-gray-filled circle

509 while the migration component locates inside the outer light-gray ring. The transition zone is 510 the middle-gray ring between them.

Figure 2. Sketch of a Gaussian filter in (a) the Fourier domain and (b) the space domain.

512 In the Fourier domain, the Gaussian filter is determined by the cutoff wavenumber, $k_{\text {tomo-max }}$ 513 and its corresponding amplitude value, $\beta . \gamma$ is the truncation value to the space-domain

514 Gaussian filter while $l$ is the radius of the Gaussian filter corresponding to $\gamma$.

Figure 3. Gradients from the experiment of the one-source-one-receiver geometry. The true model is a two-layer model, the velocities of which are $2000 \mathrm{~m} / \mathrm{s}$ for the top layer and 2200

$517 \mathrm{~m} / \mathrm{s}$ for the bottom layer. (a) The raw gradient. The blue and green crosses indicate the source 518 and receiver position. The gray dash line in (a) represents the interface of the two layers. The 519 initial model has the same geometry as the true model but the velocities are $1950 \mathrm{~m} / \mathrm{s}$ and 2145 $520 \mathrm{~m} / \mathrm{s}$ for the top and bottom layers, respectively. (b) The source wavelet, the spectrum of which 521 is flat between $3 \mathrm{~Hz}$ and $20 \mathrm{~Hz}$ shown as the dashed curve in Figures $5 \mathrm{a}$ and $5 \mathrm{~b}$. (c) The 522 wavenumber spectrum of the raw gradient. (d) The enlarged view of the central part of (c). The 523 solid blue and red circles represent the maximum wavenumber of the tomographic component, $524 k_{\text {tomo-max }}$, for $6 \mathrm{~Hz}$ and $12 \mathrm{~Hz}$, respectively. The dash blue, red and cyan circles indicate the 525 maximum wavenumber of the migration component for $6 \mathrm{~Hz}, 12 \mathrm{~Hz}$ and $24 \mathrm{~Hz}$, respectively. 
526 As we invert pressure data for velocity, the unit of the gradient is $P a^{2} \cdot s / m$, where $P a$ stands 527 for Pascal.

Figure 4. (a) The tomographic component of the gradient extracted with non-stationary smoothing frequency by frequency. (b) The wavenumber spectrum of (a).

Figure 5. Extraction of the tomographic component for the one-source-one-receiver geometry. (a) Four frequency groups used for frequency selection. The reference frequencies labelled on the horizontal axis are $1.5 \mathrm{~Hz}, 3 \mathrm{~Hz}, 6 \mathrm{~Hz}$, and $12 \mathrm{~Hz}$. The four color curves indicate the filters used for frequency selection. The dashed curve represents the spectrum of the wavelet shown in Figure 3b. (b) The same as (a) but with two frequency groups. (c) The tomographic component extracted with the four frequency groups shown in (a). (d) The wavenumber spectrum of the gradient shown in (c). (e) and (f) show the raw gradients selected by the two frequency groups. (e) corresponds to the lower frequency group while (f) is for the

538 higher frequency group. (g) The tomographic component extracted with the two frequency 539 groups shown in (b). The triangle indicates weak artifacts. (h-j) show the wavenumber spectra 540 of the gradients in (e-g), respectively. The blue and red circles represent the maximum 541 wavenumber of the tomographic component, $k_{\text {tomo-max }}$, for the reference frequencies, $6 \mathrm{~Hz}$ and $54212 \mathrm{~Hz}$, respectively.

Figure 6. Gaussian smoothing on the raw gradient shown in Figure 3a with the reference

544 frequencies of (a) $6 \mathrm{~Hz}$ and (b) $12 \mathrm{~Hz}$. (c) and (d) show the wavenumber spectra of (a) and (b), 545 respectively. The blue and red circles represent the maximum wavenumber of the tomographic 546 component, $k_{\text {tomo-max }}$, for the reference frequencies, $6 \mathrm{~Hz}$ and $12 \mathrm{~Hz}$, respectively.

Figure 7. (a) The true Marmousi model. (b) The 1D initial velocity model.

Figure 8. (a) The source wavelet and (b) its frequency spectrum. 
549 Figure 9. One shot gather of (a) the raw and (b) muted record.

550 Figure 10. The gradients of the first iteration for the background update. A multi-scale 551 strategy is applied in the inversion. The data for the first iteration are extracted by a band552 passed filter centered at $4 \mathrm{~Hz}$. The two reference frequencies used for extracting the 553 tomographic component is $2.67 \mathrm{~Hz}$ and $5.33 \mathrm{~Hz}$, the raw gradients of which are shown in (a) 554 and (c), respectively. (e) The stack of (a) and (c). (b), (d) and (f) are the counterpart of (a), (c) 555 and (e) after the non-stationary smoothing. (g) The tomographic component extracted using 556 stationary smoothing with a reference frequency of $4 \mathrm{~Hz}$, a reference velocity of $2700 \mathrm{~m} / \mathrm{s}$ and 557 a minimum opening angle, $\alpha_{\min }$, of $160^{\circ}$. (h) The same as (g) but for a reference frequency of $55812 \mathrm{~Hz}$.

Figure 11. The wavenumber spectra of the gradients shown in Figure 10. (a), (b), (e) and 560 (f) correspond to panel (a), (c), (b) and (d) of Figure 10, respectively. (c) and (d) are an enlarged 561 view of (a) and (b), respectively. The blue and red curves in (a), (c) and (e) represent the 562 maximum wavenumber of the tomographic component, $k_{\text {tomo-max }}$, of the reference frequency of $5632.67 \mathrm{~Hz}$ for the velocity of $1600 \mathrm{~m} / \mathrm{s}$ and $4000 \mathrm{~m} / \mathrm{s}$, respectively. The blue and red curves in 564 (b), (d) and (f) are the same as those in (a), (c) and (e) but for the reference frequency of 5.33 $565 \mathrm{~Hz}$.

Figure 12. Recovered Marmousi models from the 1D initial model using RWI with (a) non-stationary smoothing, stationary smoothing at the reference frequency of (b) $4 \mathrm{~Hz}$ and (c)

$56812 \mathrm{~Hz}$, the reference velocity of $2700 \mathrm{~m} / \mathrm{s}$ and the minimum opening angle, $\alpha_{\min }$, of $160^{\circ}$.

Figure 13. Recovered Marmousi models of FWI starting from (a) the initial 1D model, 570 and starting from (b-d) the RWI inverted models depicted in Figures 12 (a-c), respectively. 
571 Figure 14. Alternating display of one shot gather, comparing the records with the 572 corresponding predicted gathers from (a) the 1d initial model, (b) the recovered model of RWI 573 with non-stationary smoothing shown in Figure 12a, and (c) the recovered model of further 574 FWI shown in Figure 13b. From left to right in each panel, the recorded data are shown first 575 followed by the predicted data, and them alternating them. The dashed lines indicate the mute 576 position of the direct arrivals and refraction events.

577 Figure 15. RTM images with (a) the initial model, (b) the recovered model from RWI 578 with non-stationary smoothing shown in Figure 12a, (c) the recovered model from further FWI 579 shown in Figure 13b, and (d) the true model shown in Figure 7a.

580 Figure 16. Two common-image-gathers (CIGs) at a distance of (a-d) $2.5 \mathrm{~km}$ and (e-h) $581 \quad 8.75 \mathrm{~km}$. (a) and (e) are from the initial model. (b) and (f) are from the RWI model with non582 stationary smoothing shown in Figure 12a. (c) and (g) are from the further FWI model shown 583 in Figure 13b. (d) and (h) are from the true model shown in Figure 7a. 


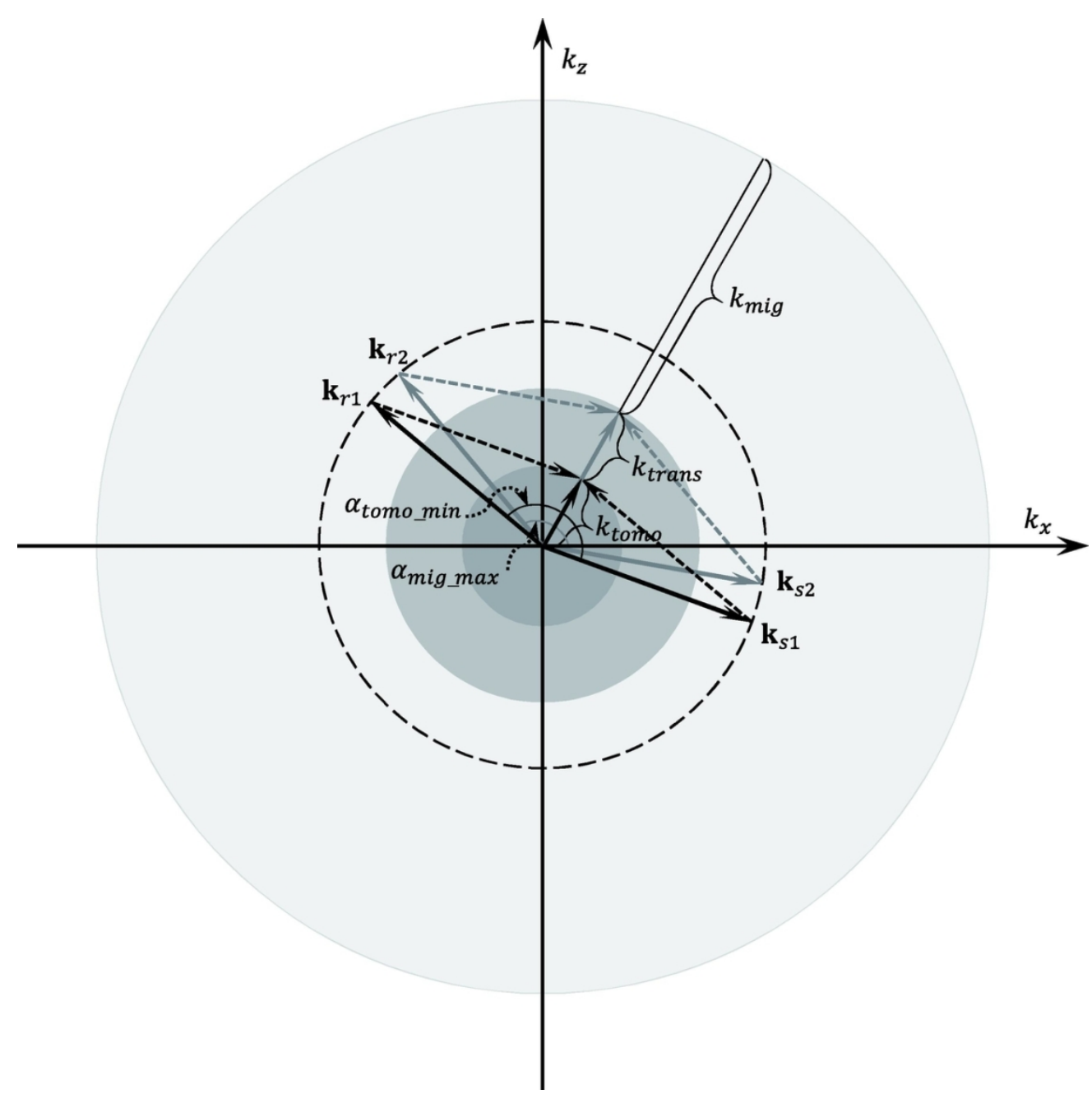

Figure 1. Schematic diagram for the relationship between opening angles and wavenumber distribution. $a_{\text {tomo_min }}$ indicates the minimum opening angle for the tomographic component while $a_{\text {mig_max }}$ indicates the maximum opening angle for the migration component. $\mathbf{k}_{\mathrm{s}}$ and $\mathbf{k}_{\mathrm{r}}$ are the wavenumber of the source and residual wavefields, respectively. They sit on the dashed circle. The tomographic component sits inside the inner dark-gray-filled circle while the migration component locates inside the outer light-gray ring. The transition zone is the middle-gray ring between them. 
2

3

4

5

6

ot

8

3

0

氕 1

곤 2

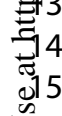

96

97

్ㅡㅇ 8

ज9

20

资2

3 (a)

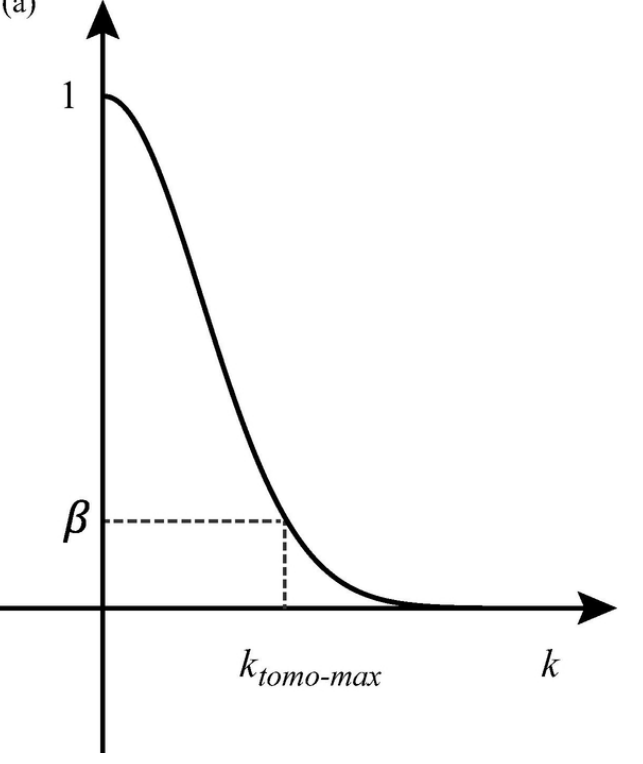

(b)

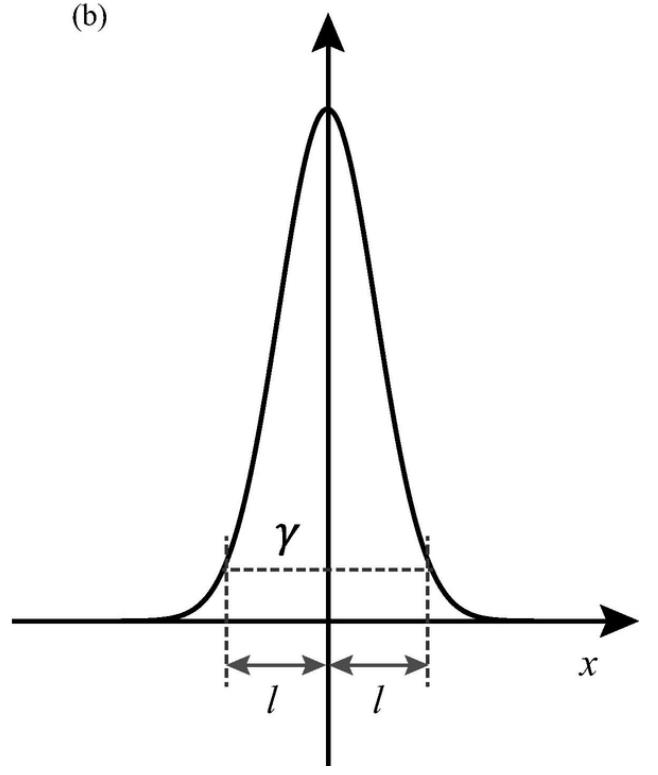

Figure 2. Sketch of a Gaussian filter in (a) the Fourier domain and (b) the space domain. In the Fourier domain, the Gaussian filter is determined by the cutoff wavenumber, $k_{\text {tomo }}$ max and its corresponding amplitude value, $\beta . \gamma$ is the truncation value to the space-domain Gaussian filter while $/$ is the radius of the Gaussian filter corresponding to $y$. 
(a) Distance $(\mathrm{km})$

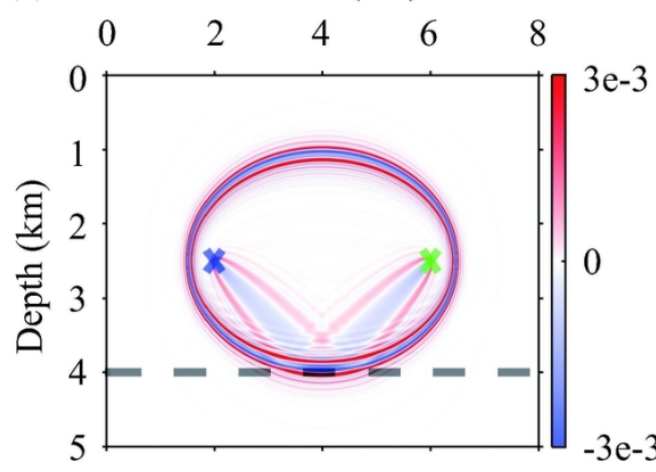

(c)

$$
\begin{array}{ccccc} 
& \multicolumn{3}{c}{\mathrm{kx}(1 / \mathrm{m})} & \times 10^{-2} \\
-2 & -1 & 0 & 1 & 2
\end{array}
$$

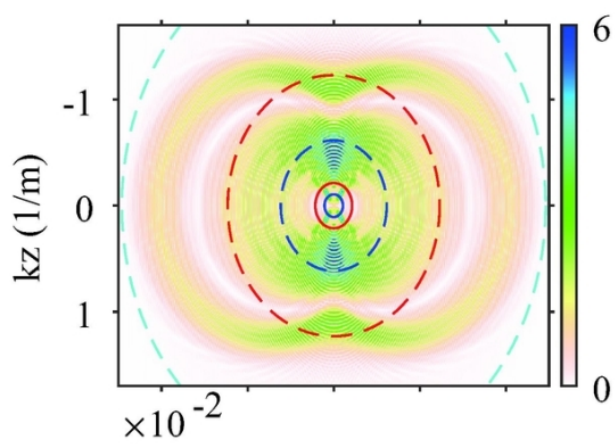

(b)

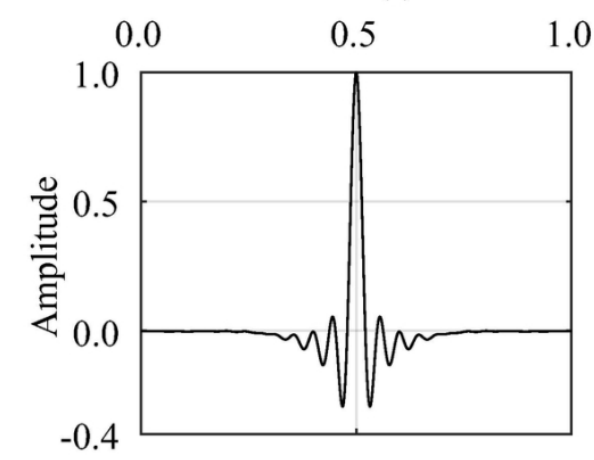

(d)

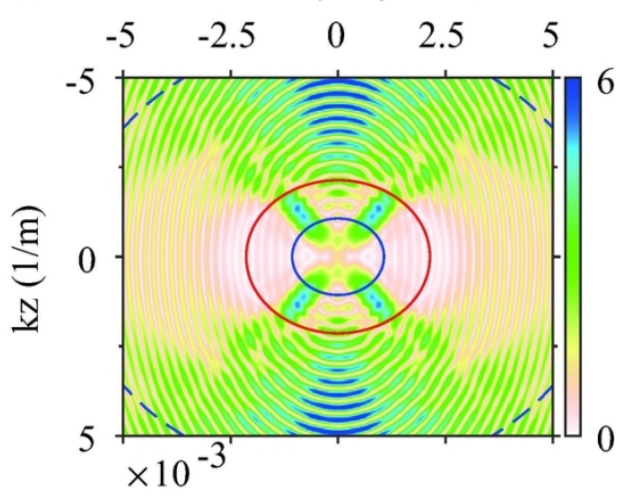

Figure 3. Gradients from the experiment of the one-source-one-receiver geometry. The true model is a twolayer model, the velocities of which are $2000 \mathrm{~m} / \mathrm{s}$ for the top layer and $2200 \mathrm{~m} / \mathrm{s}$ for the bottom layer. (a) The raw gradient. The blue and green crosses indicate the source and receiver position. The gray dash line in (a) represents the interface of the two layers. The initial model has the same geometry as the true model but the velocities are $1950 \mathrm{~m} / \mathrm{s}$ and $2145 \mathrm{~m} / \mathrm{s}$ for the top and bottom layers, respectively. (b) The source wavelet, the spectrum of which is flat between $3 \mathrm{~Hz}$ and $20 \mathrm{~Hz}$ shown as the dashed curve in Figures $5 \mathrm{a}$ and 5b. (c) The wavenumber spectrum of the raw gradient. (d) The enlarged view of the central part of (c). The solid blue and red circles represent the maximum wavenumber of the tomographic component, $k_{\text {tomo_max, }}$ for $6 \mathrm{~Hz}$ and $12 \mathrm{~Hz}$, respectively. The dash blue, red and cyan circles indicate the maximum wavenumber of the migration component for $6 \mathrm{~Hz}, 12 \mathrm{~Hz}$ and $24 \mathrm{~Hz}$, respectively. As we invert pressure data for velocity, the unit of the gradient is $\mathrm{Pa}^{2} \cdot \mathrm{s} / \mathrm{m}$, where $\mathrm{Pa}$ stands for Pascal. 
(a)

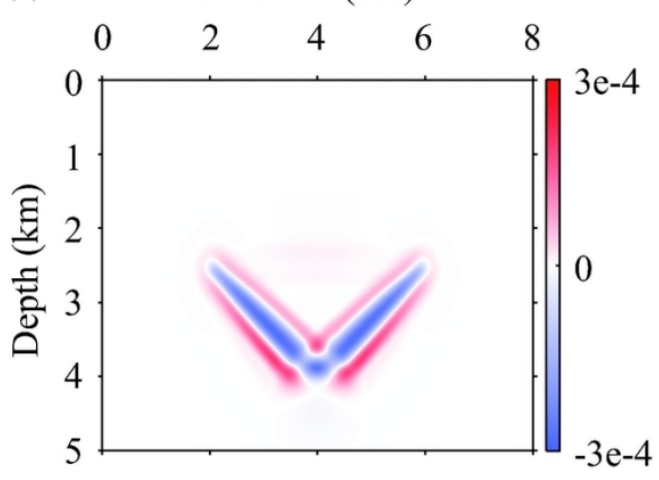

(b) $\mathrm{kx}(1 / \mathrm{m}) \times 10^{-3}$

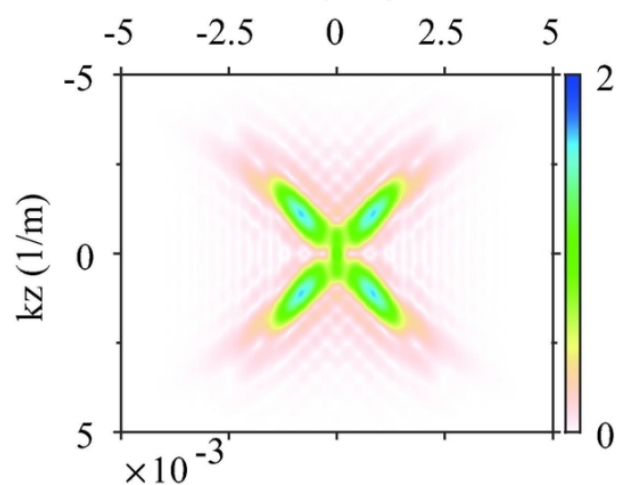

Figure 4. (a) The tomographic component of the gradient extracted with non-stationary smoothing frequency by frequency. (b) The wavenumber spectrum of (a).

$109 \times 46 \mathrm{~mm}(300 \times 300 \mathrm{DPI})$ 
(d)
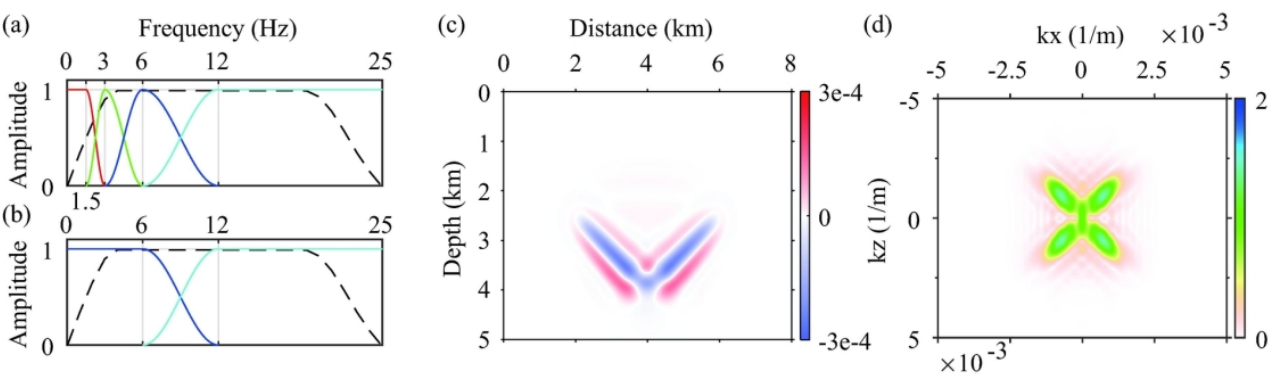

(e)

Distance $(\mathrm{km})$

(f)

f) Distance $(\mathrm{km})$

(g)

g) Distance $(\mathrm{km})$
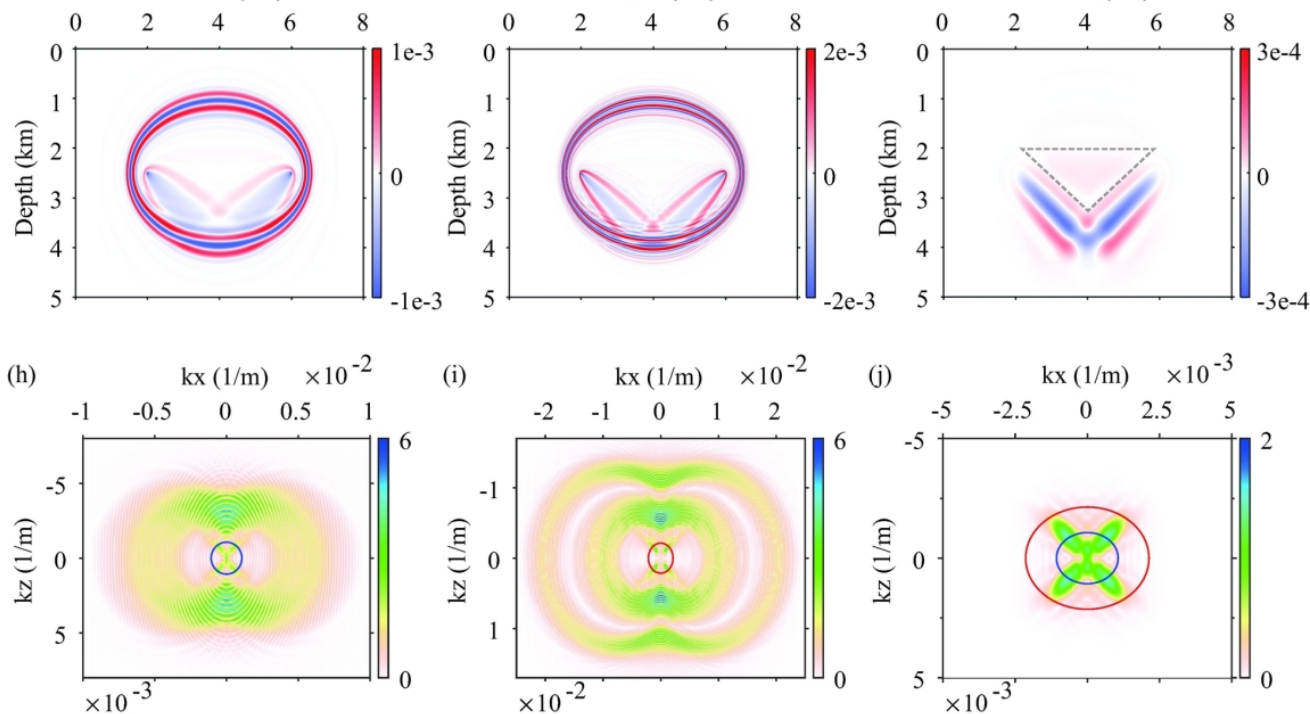

(j) $\quad \mathrm{kx}(1 / \mathrm{m}) \times 10^{-3}$

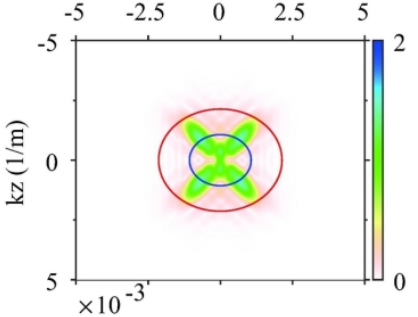

Figure 5. Extraction of the tomographic component for the one-source-one-receiver geometry. (a) Four frequency groups used for frequency selection. The reference frequencies labelled on the horizontal axis are $1.5 \mathrm{~Hz}, 3 \mathrm{~Hz}, 6 \mathrm{~Hz}$, and $12 \mathrm{~Hz}$. The four color curves indicate the filters used for frequency selection. The dashed curve represents the spectrum of the wavelet shown in Figure 3b. (b) The same as (a) but with two frequency groups. (c) The tomographic component extracted with the four frequency groups shown in (a). (d) The wavenumber spectrum of the gradient shown in (c). (e) and (f) show the raw gradients selected by the two frequency groups. (e) corresponds to the lower frequency group while (f) is for the higher frequency group. (g) The tomographic component extracted with the two frequency groups shown in (b). The triangle indicates weak artifacts. ( $h-j)$ show the wavenumber spectra of the gradients in (e-g), respectively. The blue and red circles represent the maximum wavenumber of the tomographic component, $k_{\text {tomo_max }}$, for the reference frequencies, $6 \mathrm{~Hz}$ and $12 \mathrm{~Hz}$, respectively. 
(a) Distance (km)

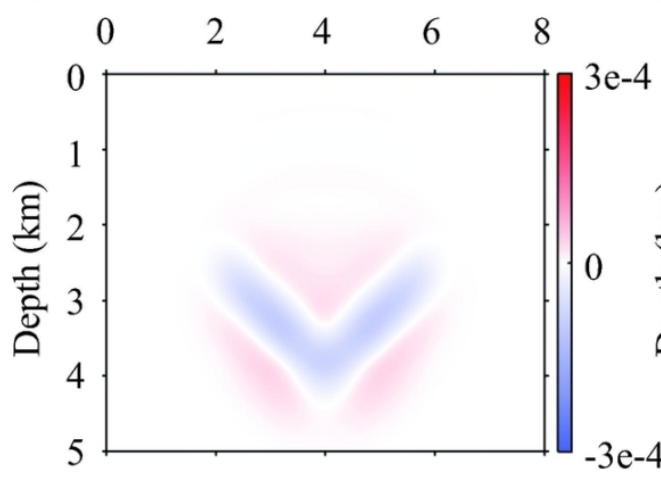

(b)

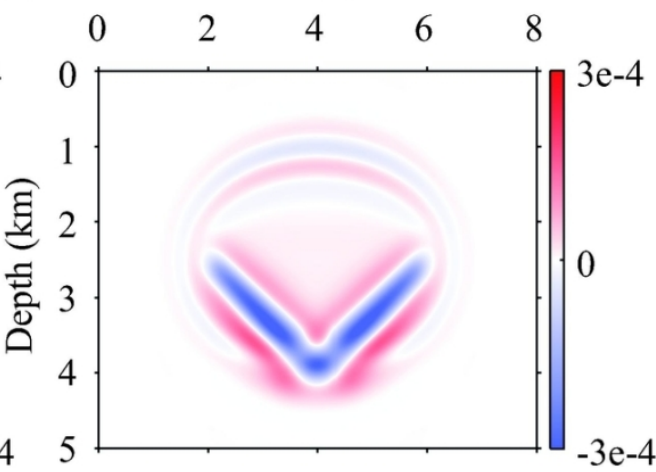

(c)

$\mathrm{kx}(1 / \mathrm{m}) \times 10^{-3}$
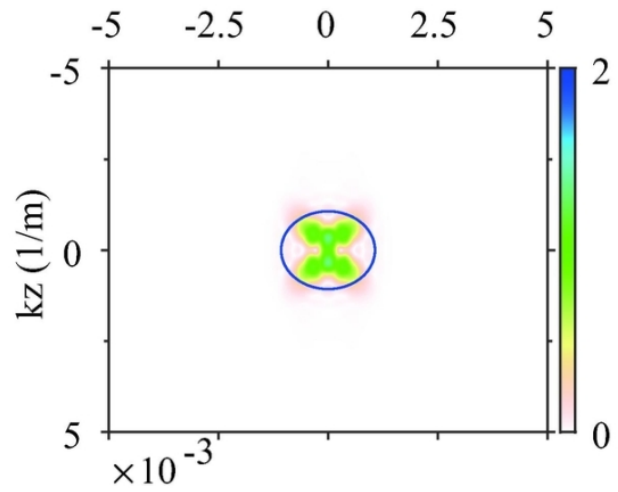

(d)

d) $\mathrm{kx}(1 / \mathrm{m}) \times 10^{-3}$

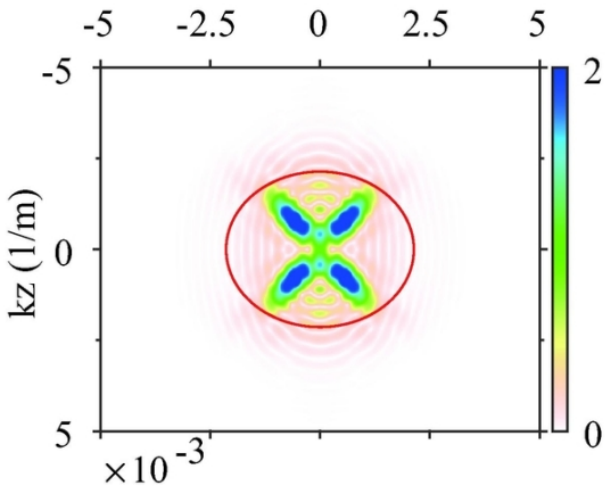

Figure 6. Gaussian smoothing on the raw gradient shown in Figure 3a with the reference frequencies of (a) 6 $\mathrm{Hz}$ and (b) $12 \mathrm{~Hz}$. (c) and (d) show the wavenumber spectra of (a) and (b), respectively. The blue and red circles represent the maximum wavenumber of the tomographic component, $k_{\text {tomo_max }}$ for the reference frequencies, $6 \mathrm{~Hz}$ and $12 \mathrm{~Hz}$, respectively. 


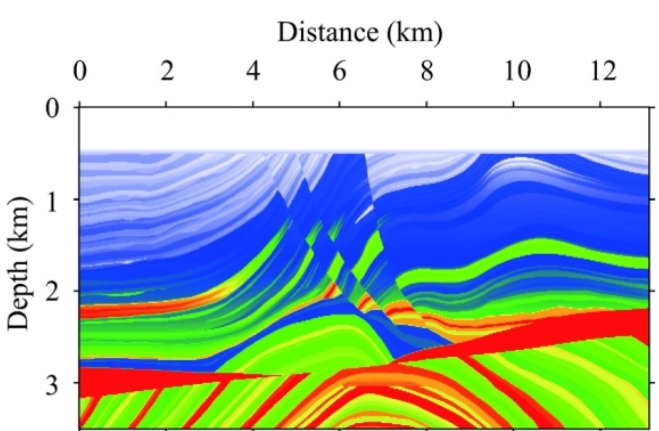

(a)

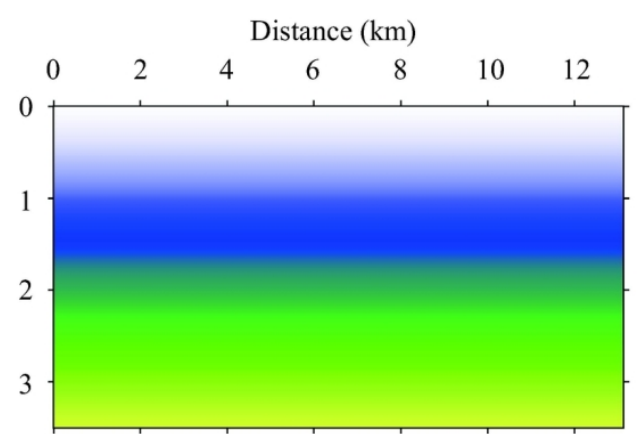

(b)

Figure 7. (a) The true Marmousi model. (b) The 1D initial velocity model.

$141 \times 60 \mathrm{~mm}(300 \times 300 \mathrm{DPI})$ 


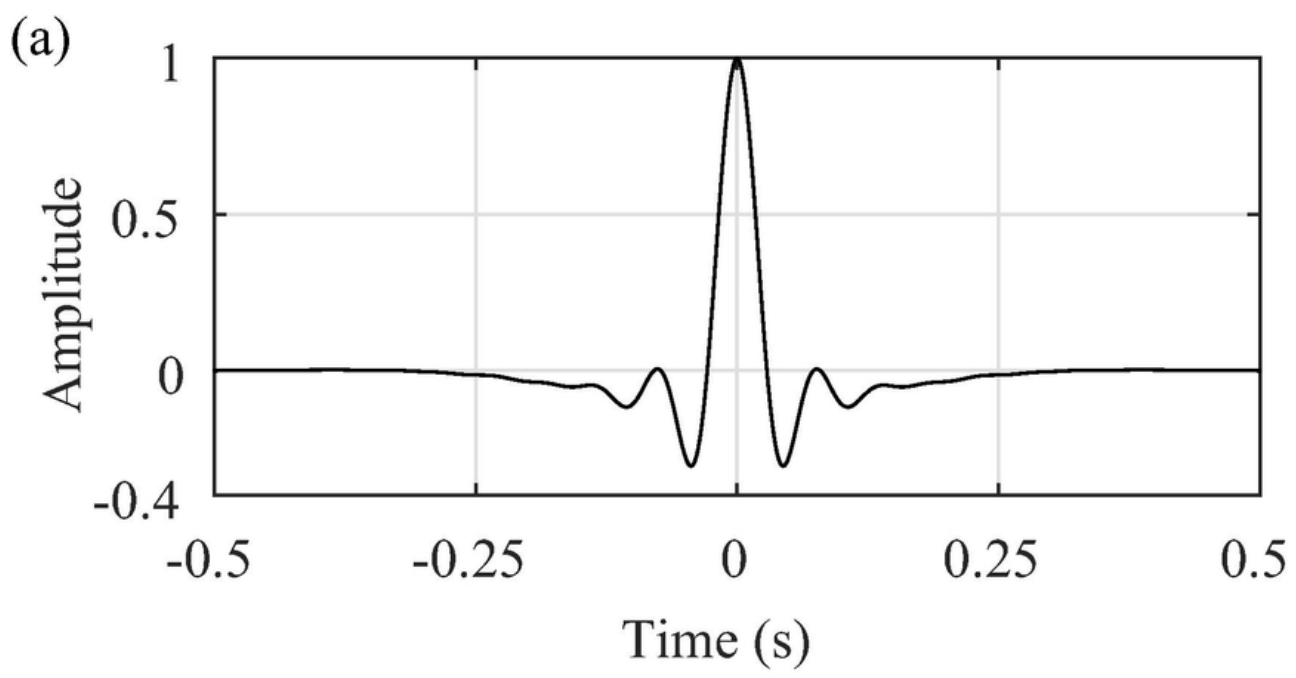

(b)

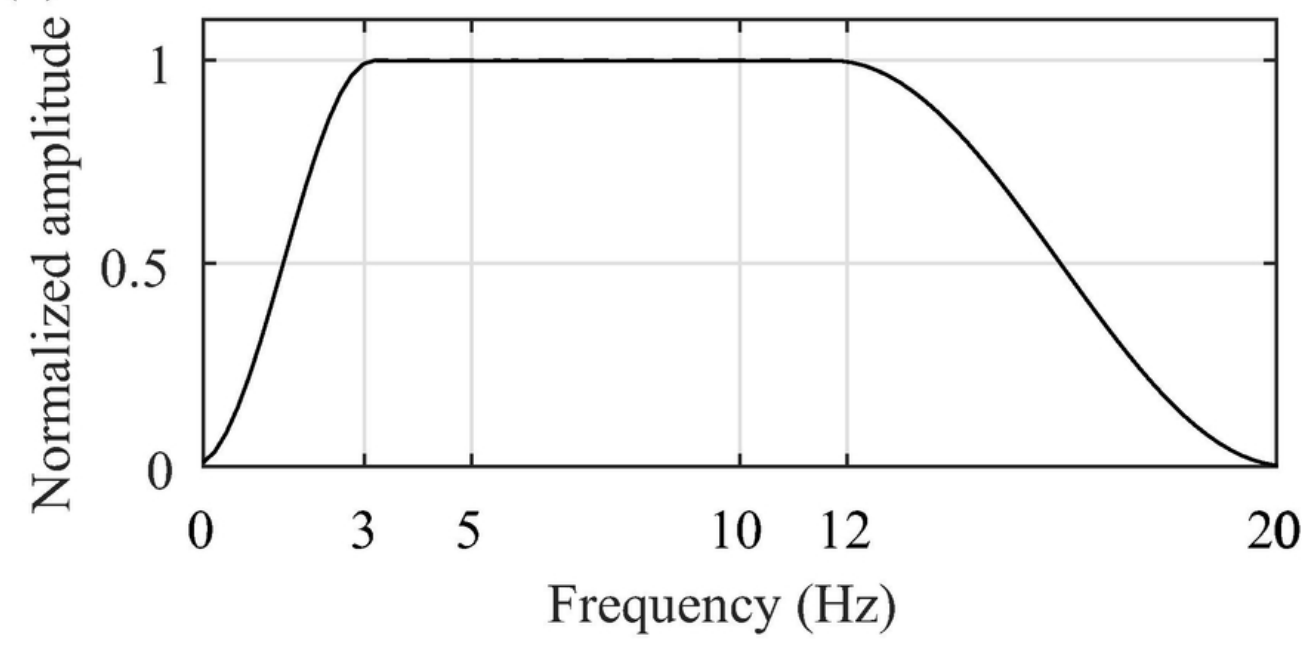

Figure 8. (a) The source wavelet and (b) its frequency spectrum.

$74 \times 78 \mathrm{~mm}(300 \times 300$ DPI $)$ 


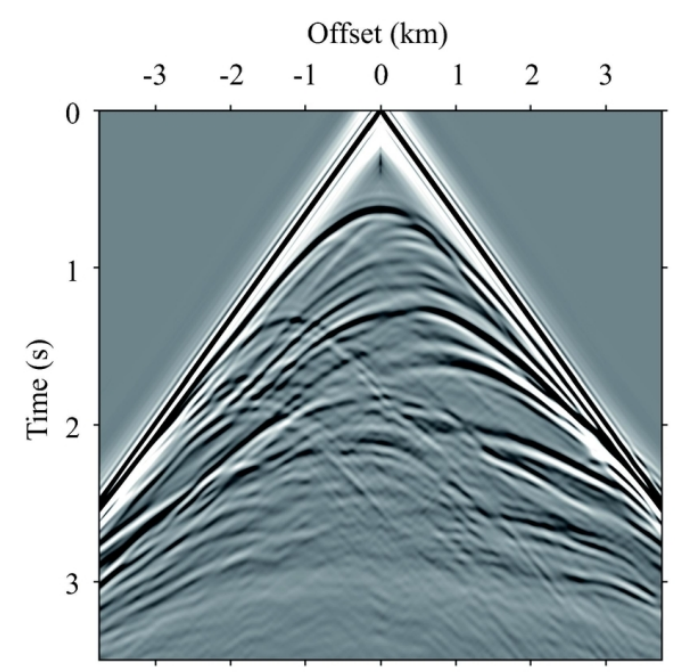

(a)

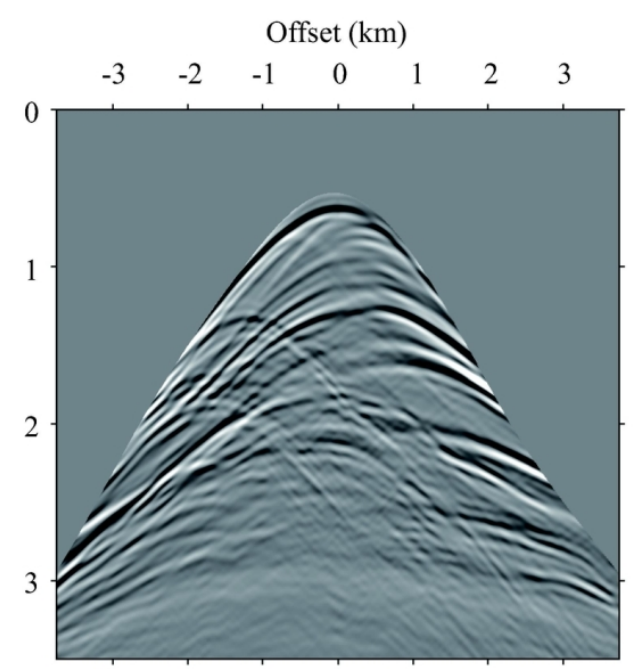

(b)

Figure 9. One shot gather of (a) the raw and (b) muted record.

$136 \times 73 \mathrm{~mm}(300 \times 300 \mathrm{DPI})$ 

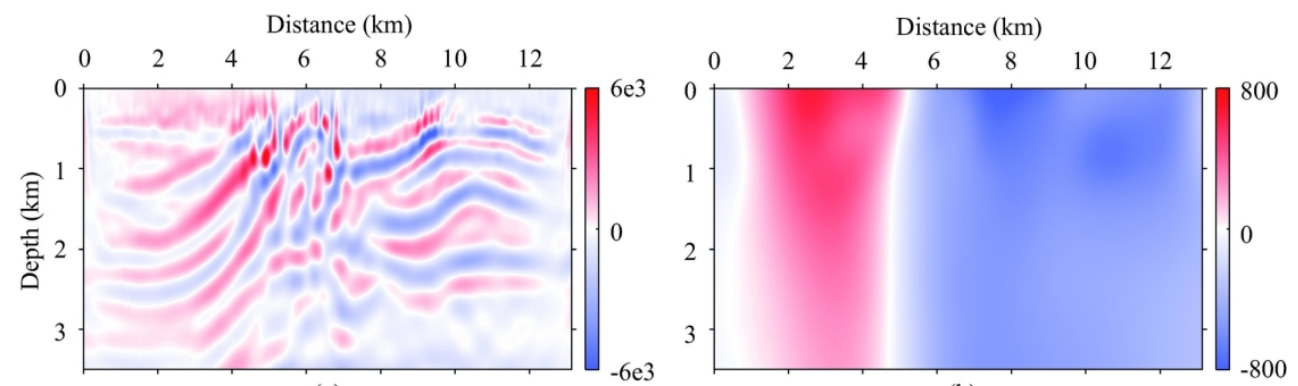

(a)
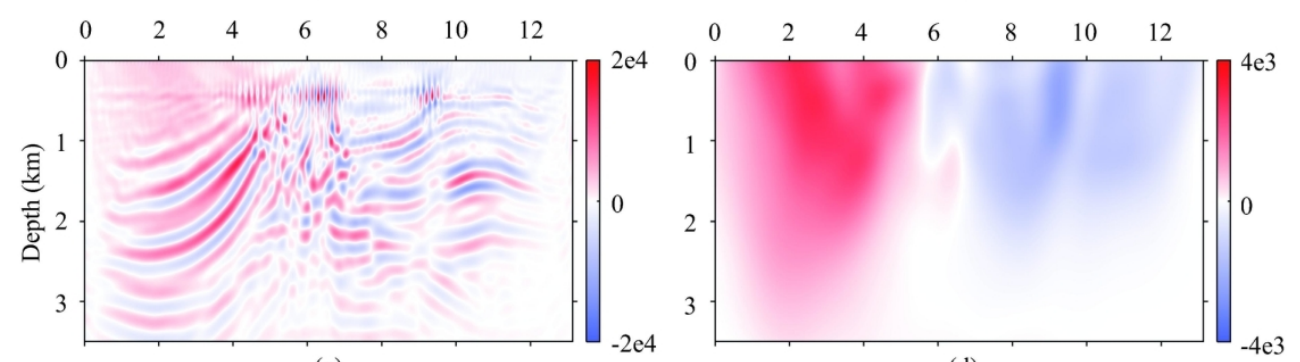

(c)

(d)

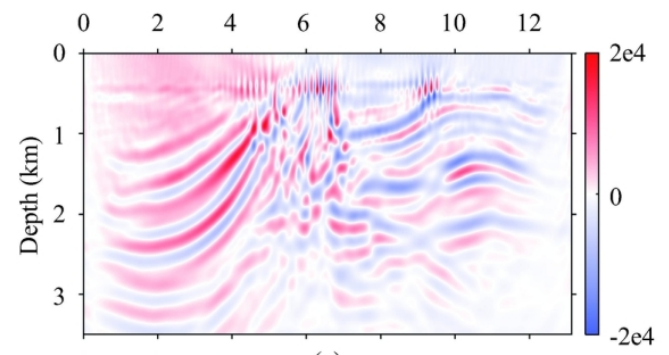

(e)

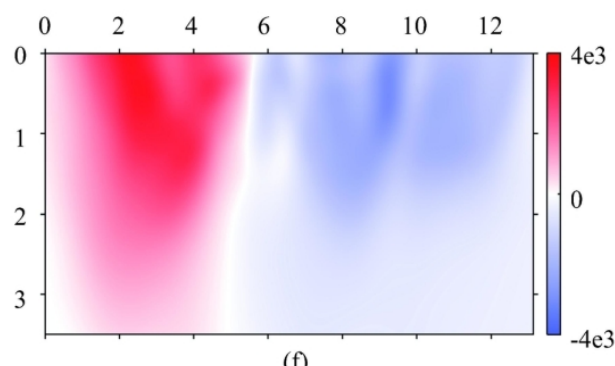

(f)

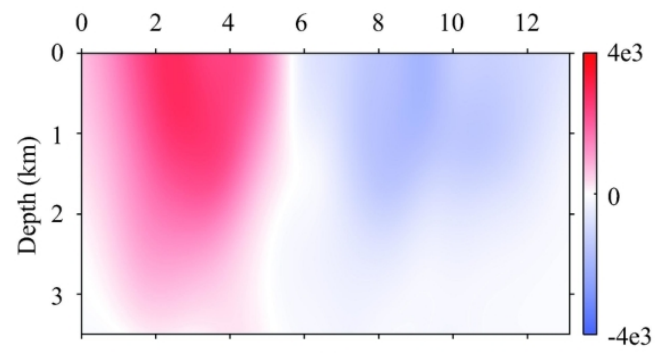

(g)

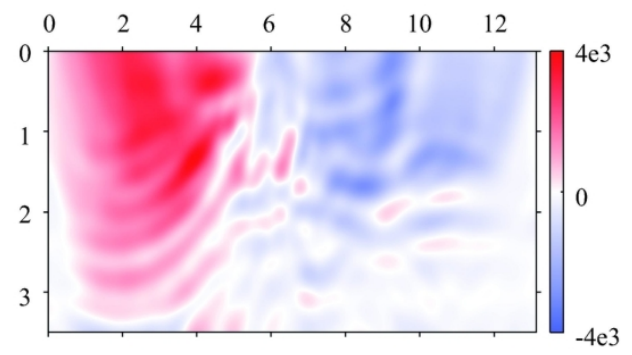

(h)

Figure 10. The gradients of the first iteration for the background update. A multi-scale strategy is applied in the inversion. The data for the first iteration are extracted by a band-passed filter centered at $4 \mathrm{~Hz}$. The two reference frequencies used for extracting the tomographic component is $2.67 \mathrm{~Hz}$ and $5.33 \mathrm{~Hz}$, the raw gradients of which are shown in (a) and (c), respectively. (e) The stack of (a) and (c). (b), (d) and (f) are the counterpart of (a), (c) and (e) after the non-stationary smoothing. (g) The tomographic component extracted using stationary smoothing with a reference frequency of $4 \mathrm{~Hz}$, a reference velocity of $2700 \mathrm{~m} / \mathrm{s}$ and a minimum opening angle, $a_{\min }$, of $160^{\circ}$. (h) The same as ( $\mathrm{g}$ ) but for a reference frequency of $12 \mathrm{~Hz}$. 
(a)

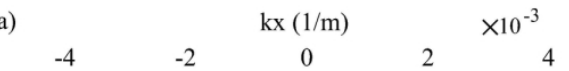

(b)
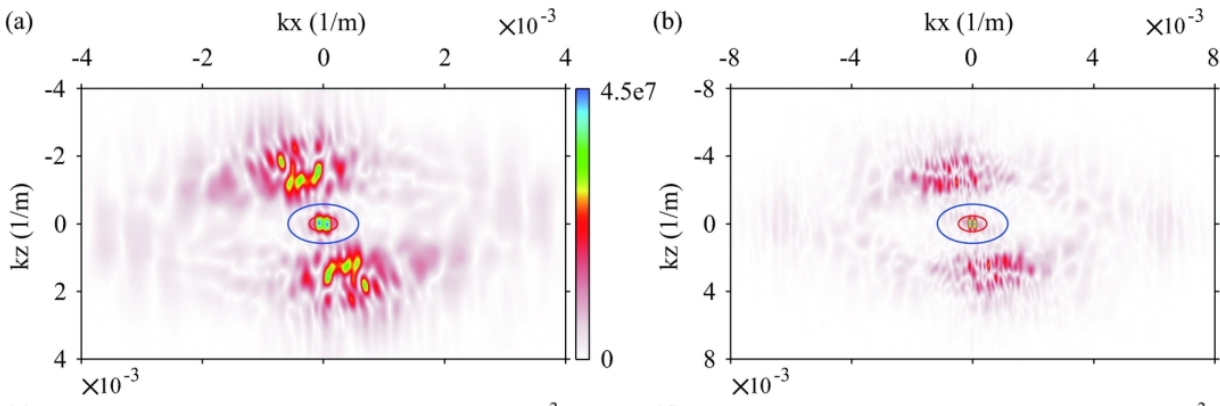

(c)
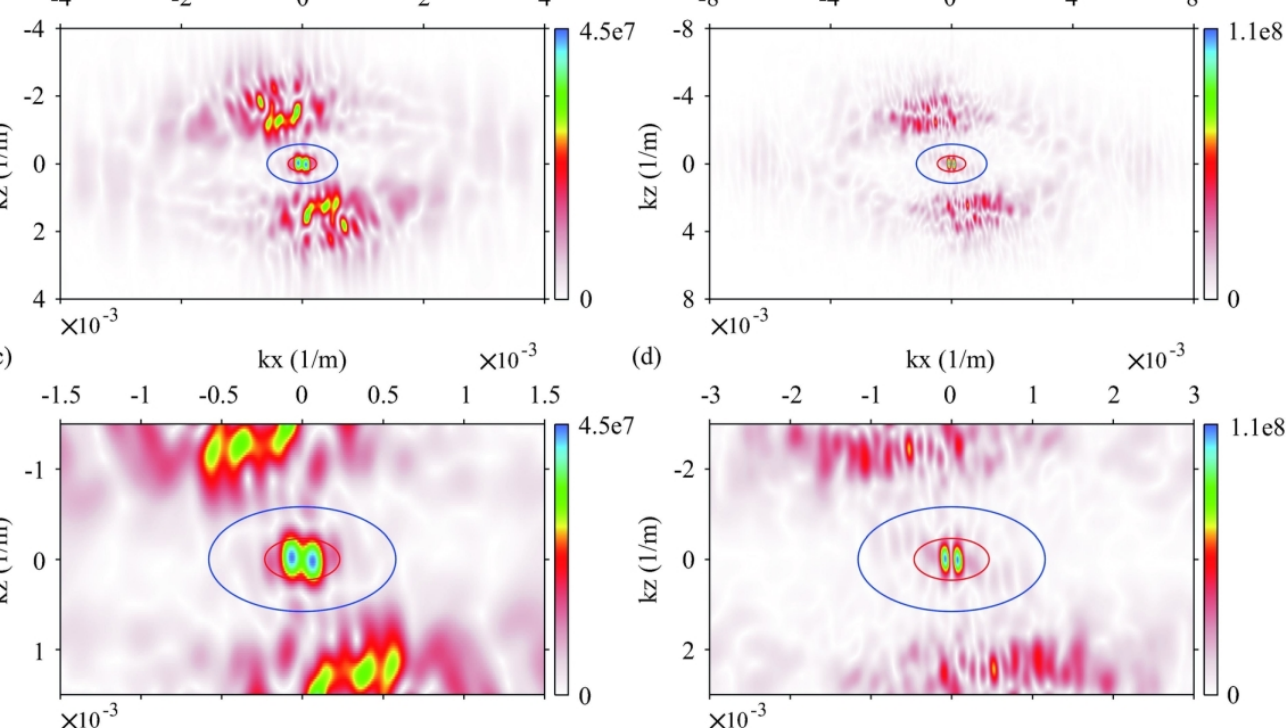

(d)
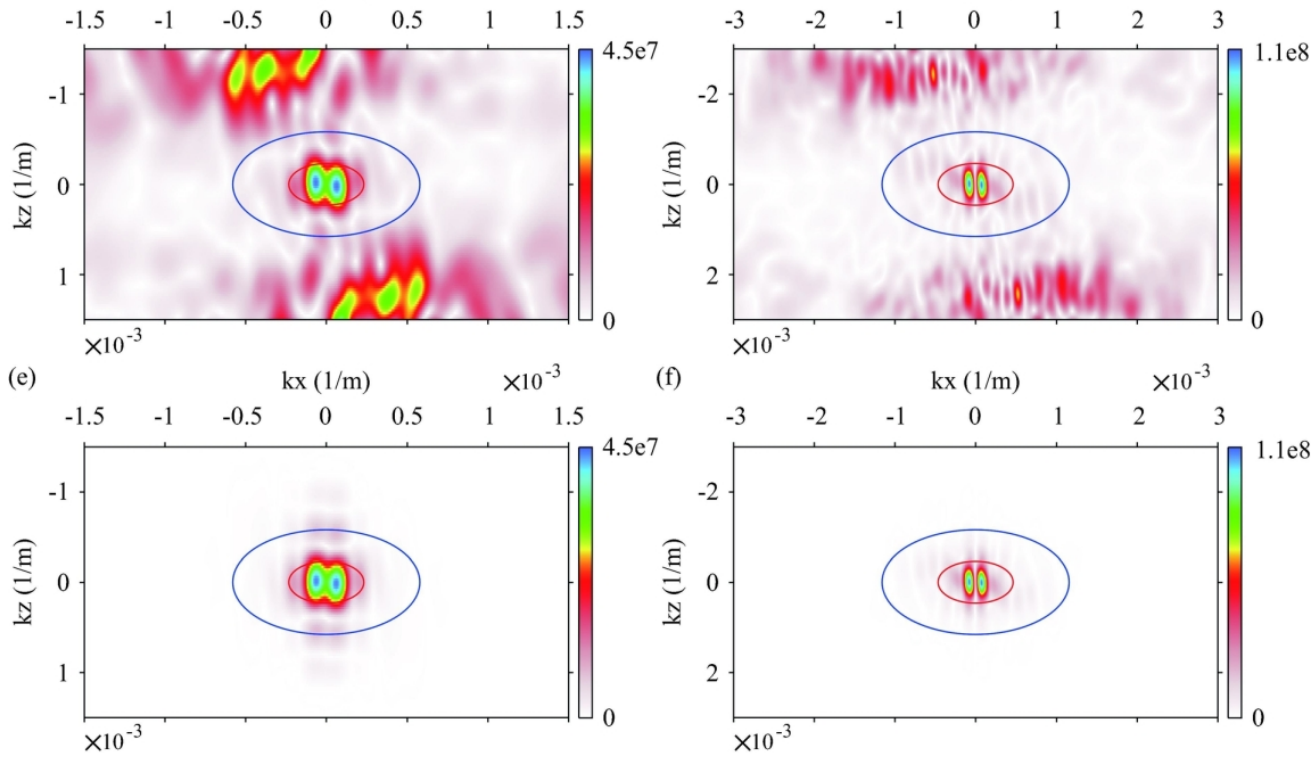

Figure 11. The wavenumber spectra of the gradients shown in Figure 10. (a), (b), (e) and (f) correspond to panel (a), (c), (b) and (d) of Figure 10, respectively. (c) and (d) are an enlarged view of (a) and (b), respectively. The blue and red curves in (a), (c) and (e) represent the maximum wavenumber of the

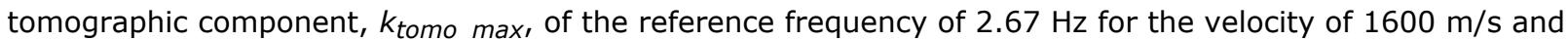
$4000 \mathrm{~m} / \mathrm{s}$, respectively. The blue and red curves in (b), (d) and (f) are the same as those in (a), (c) and (e) but for the reference frequency of $5.33 \mathrm{~Hz}$.

$161 \times 147 \mathrm{~mm}(300 \times 300 \mathrm{DPI})$ 

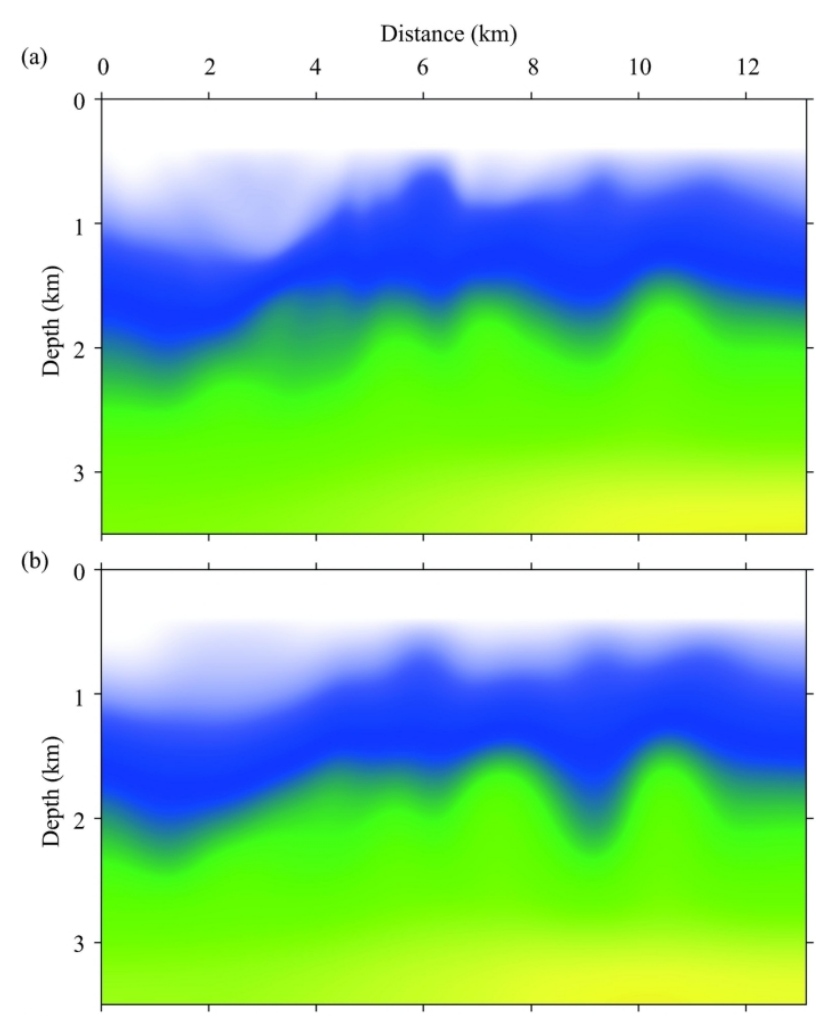

(c)

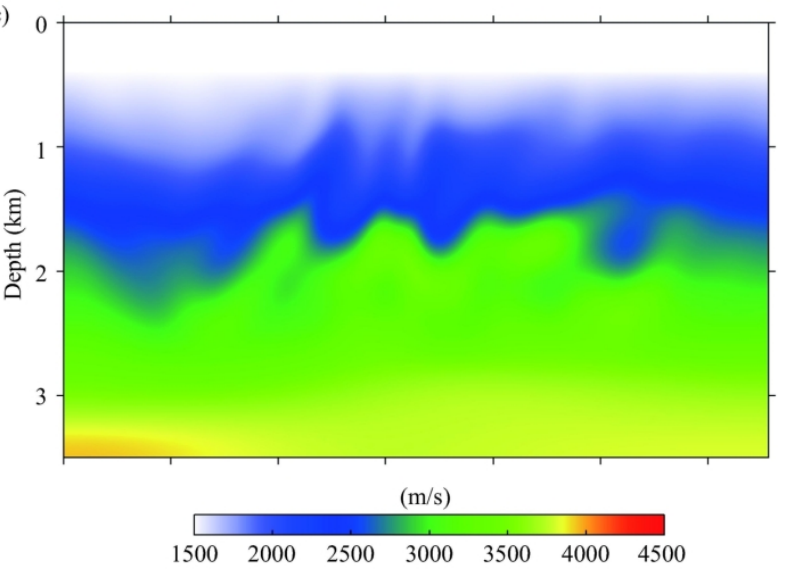

Figure 12. Recovered Marmousi models from the 1D initial model using RWI with (a) non-stationary smoothing, stationary smoothing at the reference frequency of (b) $4 \mathrm{~Hz}$ and (c) $12 \mathrm{~Hz}$, the reference velocity of $2700 \mathrm{~m} / \mathrm{s}$ and the minimum opening angle, $a_{\min }$, of $160^{\circ}$. 


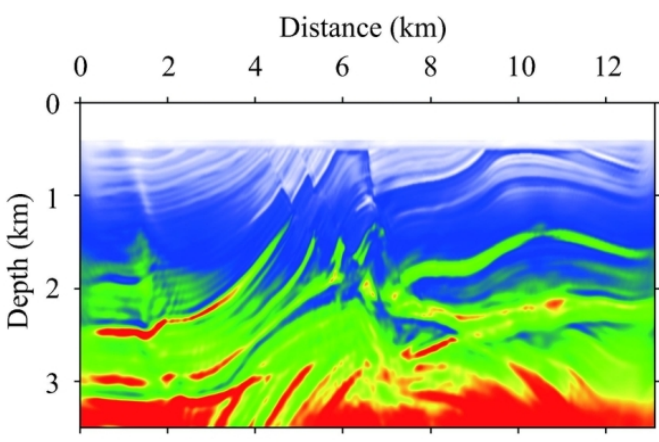

(a)

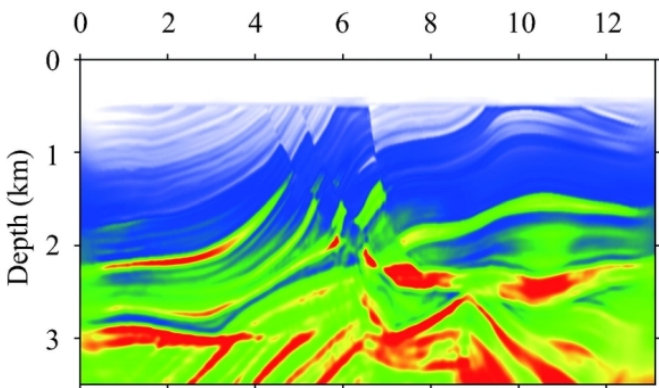

(c)

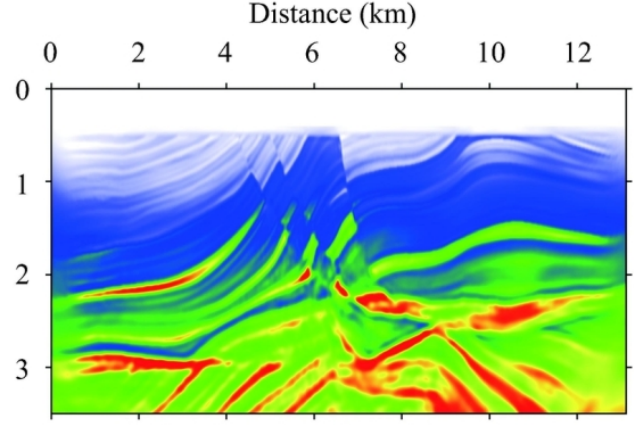

(b)

Figure 13. Recovered Marmousi models of FWI starting from (a) the initial 1D model, and starting from (b-d) the RWI inverted models depicted in Figures $12(\mathrm{a}-\mathrm{c})$, respectively. 


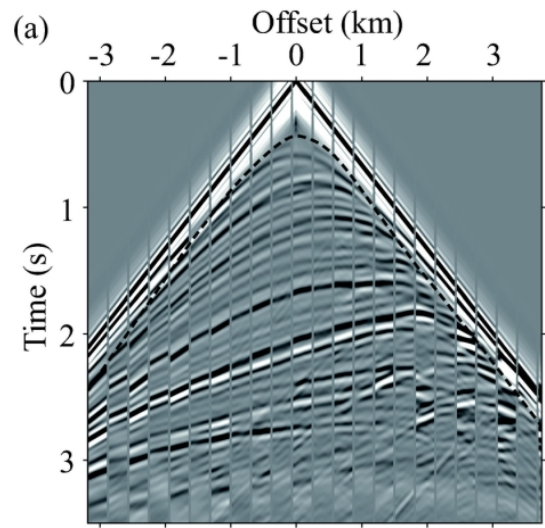

(b) $\begin{array}{rrrrrrr}-3 & -2 & -1 & 0 & 1 & 2 & 3\end{array}$

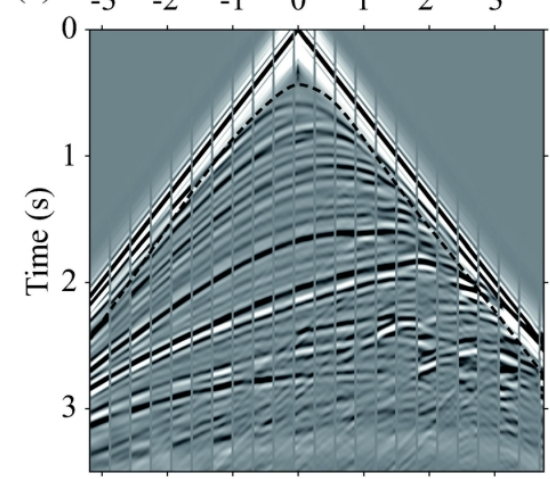

(c) $\begin{array}{rrrrrrr}-3 & -2 & -1 & 0 & 1 & 2 & 3\end{array}$

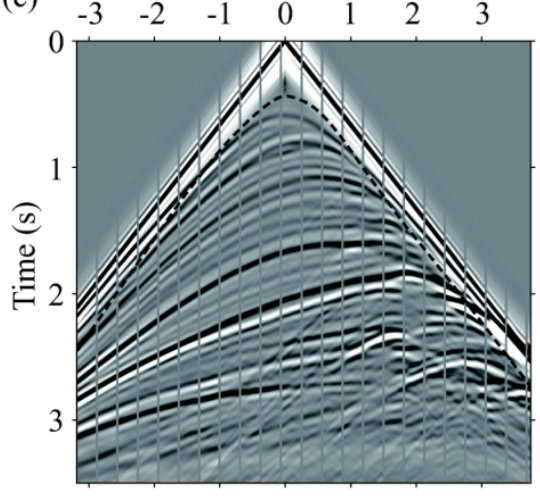

igure 14. Alternating display of one shot gather, comparing the records with the corresponding predicted gathers from (a) the 1d initial model, (b) the recovered model of RWI with non-stationary smoothing shown in Figure 12a, and (c) the recovered model of further FWI shown in Figure 13b. From left to right in each panel, the recorded data are shown first followed by the predicted data, and them alternating them. The dashed lines indicate the mute position of the direct arrivals and refraction events.

$$
70 \times 203 \mathrm{~mm}(300 \times 300 \text { DPI })
$$




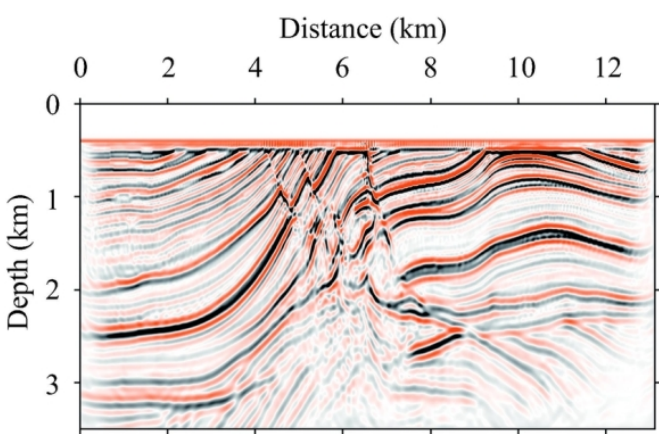

(a)

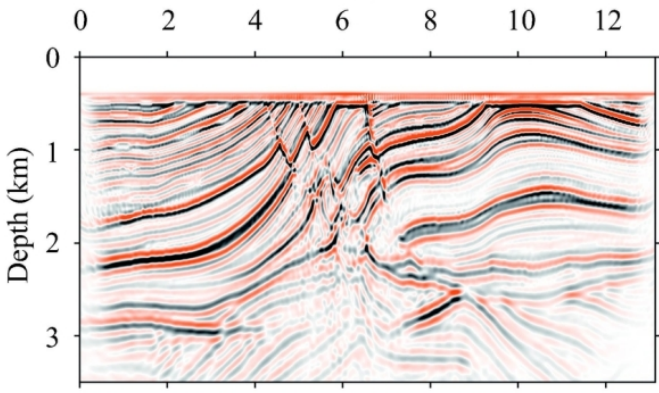

(c)

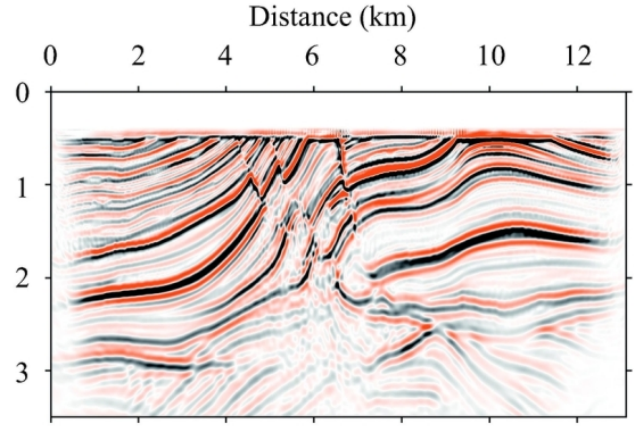

(b)

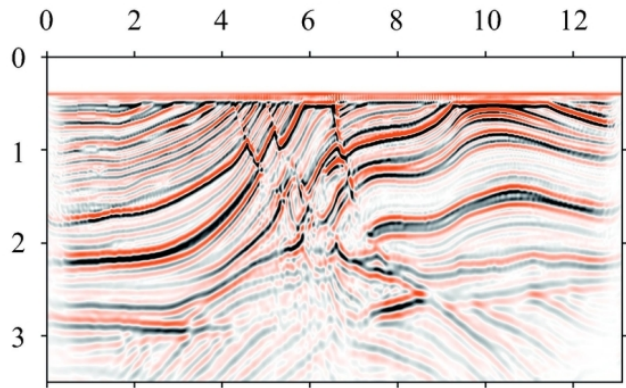

(d)

Figure 15. RTM images with (a) the initial model, (b) the recovered model from RWI with non-stationary smoothing shown in Figure 12a, (c) the recovered model from further FWI shown in Figure 13b, and (d) the true model shown in Figure $7 a$.

$140 \times 98 \mathrm{~mm}(300 \times 300 \mathrm{DPI})$ 


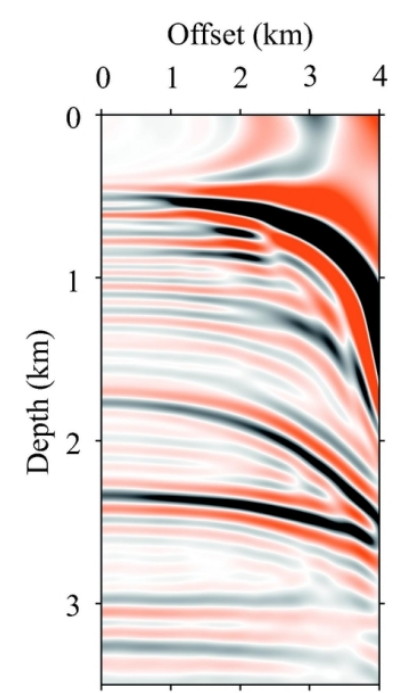

(a)

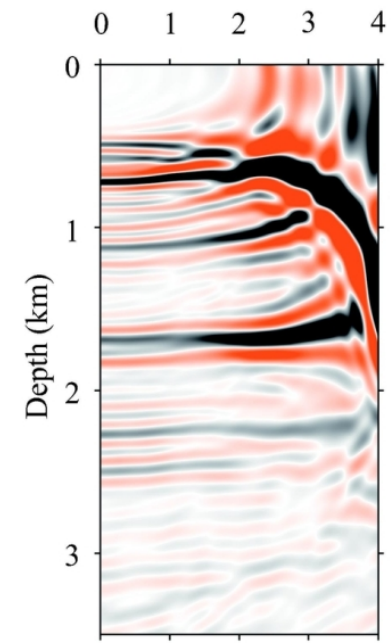

(e)

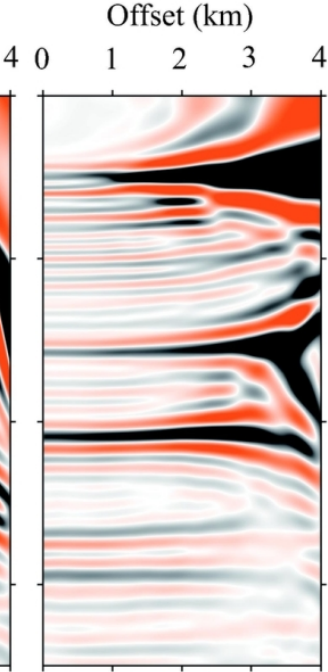

(b)

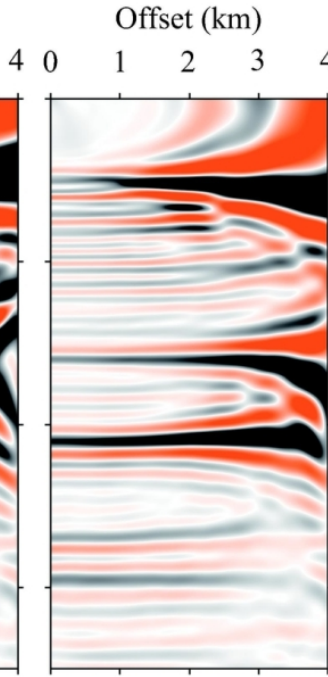

(c)

\section{$\begin{array}{lllrrr}4 & 0 & 1 & 2 & 3 & 4\end{array}$}

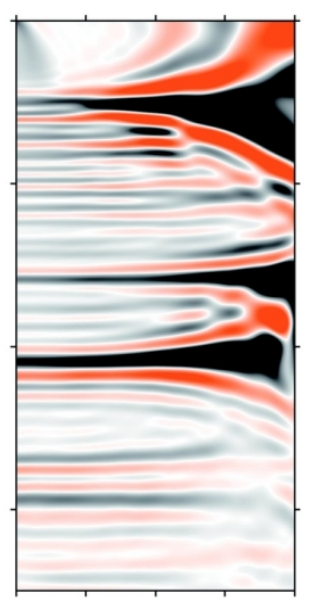

(d)

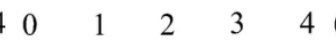

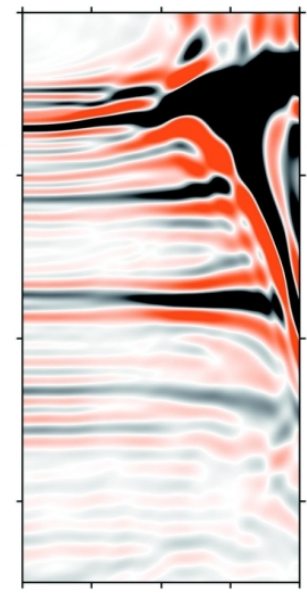

(f)

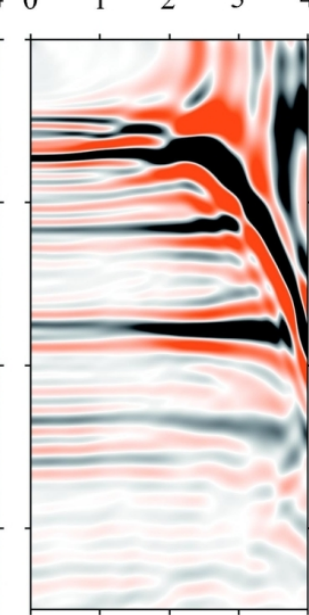

(g)

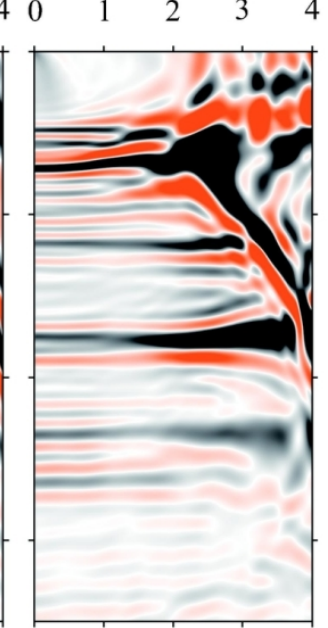

(h)

Figure 16. Two common-image-gathers (CIGs) at a distance of (a-d) $2.5 \mathrm{~km}$ and (e-h) $8.75 \mathrm{~km}$. (a) and (e) are from the initial model. (b) and (f) are from the RWI model with non-stationary smoothing shown in Figure 12a. (c) and (g) are from the further FWI model shown in Figure 13b. (d) and (h) are from the true model shown in Figure 7a.

$131 \times 143 \mathrm{~mm}(300 \times 300 \mathrm{DPI})$ 
DATA AND MATERIALS AVAILABILITY

Data associated with this research are available and can be obtained by contacting the corresponding author. 